\title{
State-Dependent Probability Distributions in Non Linear Rational Expectations Models*
}

Jean Barthélemy

Magali Marx

March 2013

\begin{abstract}
In this paper, we solve a large class of non-linear rational expectations models with regime switching, i.e. recurring shifts in parameters. The regime-switches and the shocks may follow state-dependent probability distributions. First, we provide sufficient conditions for the existence of a unique stable equilibrium using a perturbation approach. Then, we provide Taylor expansions of the solution in subclasses of models. Finally, we put forward that state-dependent fluctuations of transition probabilities can substantially alter the equilibrium dynamics through economic agents' expectations. We illustrate these modifications in the context of a Fisherian model of inflation and a NewKeynesian model in which monetary policy switches endogenously between a passive and an active reaction to inflation.
\end{abstract}

Keywords: Regime switching, DSGE, indeterminacy, monetary policy, perturbation methods.

JEL: E32, E43

${ }^{*}$ The views expressed in this paper do not necessarily reflect the opinion of the Banque de France. We thank P. Antipa, L. Benati, J. Benhabib, F. Bilbiie, A. Braun, F. Canova, E. Challe, G. Cléaud, B. Diba, S. Dubecq, M. Eichenbaum, C. Hellwig, R. Farmer, M. Giannoni, M. Juillard, E. Leeper, O. Loisel, E. Mendoza, X. Ragot, T. Sargent, J. Suda, F. Velde, D. Waggoner, C. Walsh, M. Woodford and T. Zha for their very helpful comments. We are also grateful for comments from seminar participants at the 2010 and 2011 International Conference on Computing in Economics and Finance, the Federal Reserve Bank of Atlanta , the Federal Reserve Bank of Chicago, the 2011 EEA-ESEM conference and the 2012 T2M Conference. All remaining errors are ours.

Barthélemy: Monetary Policy Research Division, Banque de France, 39 rue Croix-des-Petits-Champs, 75001 Paris, (e-mail: jean.barthelemy@banque-france.fr); Marx: Department of Economics, Sciences Po, 28 rue des Saints Pères, 75007 Paris, and Banque de France (e-mail: magali.marx@sciences-po.org) 


\section{Introduction}

In this paper, we consider a class of non-linear discrete-time rational expectations models with discrete jump processes, describing regime switching, and continuous stochastic processes, the usual "shocks". Both processes can follow state-dependent probability distributions meaning that the distribution of shocks as well as the probabilities of regime switches may depend on the state of the economy. We find sufficient determinacy conditions, i.e. conditions ensuring the existence of a unique stable solution, and provide the Taylor expansion of the solution. We apply our methodology to the Fisherian model of inflation determination and a NewKeynesian model with monetary policy switches. We show that state-dependent fluctuations of transition probabilities can substantially alter the equilibrium dynamics through economic agents' expectations.

A substantial line of empirical studies challenges the common assumption of economic agents' time-invariant behavior (Clarida et al., 2000). Recent papers (e.g., Svennson and Williams, 2009; Farmer et al., 2009b) thus relax the assumption of time-invariant behavior by introducing recurring shifts in structural parameters ; yet, a common approach remains that changes in policy behavior are exogenous.

However, there is a wide range of empirical (Kim et al., 2003) and theoretical (e.g. Mendoza and Yue, 2011, for defaults) reasons to believe that regime switches may be influenced by macroeconomic fluctuations or, more generally, that they are endogenous to the model's economic outcomes. Thus, this paper addresses this issue and considers models where shocks and regime switches have state-dependent distributions.

To solve these models, we extend the perturbation approach to state-dependent probability distributions. Using such an approach, Woodford (1986) infers the stability properties of non-linear stochastic models from their linearized counterpart assuming "small" shocks. In the same spirit, we link the stability properties of the endogenous regime switching to the exogenous regime switching under the assumption of weak sensitivity of transition probabilities to state variables and small shocks. This general result fills the gap between the non-linear endogenous regime switching models and existing results on linear regime-switching models (see for instance Davig and Leeper, 2007; Farmer et al., 2009a; Cho, 2009; Barthélemy and Marx, 2012).

Our contributions are threefold.

First, we prove that the existence and the uniqueness of a bounded solution in this class of models rely on the existence of a unique solution for a simplified, linear model with exogenous regime switching. We do that by applying the Implicit Function Theorem in a context of state-dependent probability distributions (Theorem 1).

Second, we apply this result to two distinct cases. The first case considers models in which shocks follow a state-dependent probability density function but no regime switching. Proposition 1 extends Woodford (1986) results to this specific context. When shocks are small enough, the state-dependence does not alter the determinacy conditions and only modifies the second-order expansion of the solution. The second case comprises forward-looking models with state-dependent transition probabilities of switching across regimes. Based on 
Barthélemy and Marx (2012), we characterize determinacy conditions for this class of models (Proposition 2) and provide first- and second-order Taylor expansions of the solution (Proposition 3). The state-dependence affects first-order terms only if the steady-states differ across regimes, otherwise the endogeneity has an impact at second-order only.

Third, we highlight three effects peculiar to the endogenous nature of regime switching by applying our method to two standard monetary models: a Fisherian and a new-Keynesian model. In both models, we assume that the reaction to inflation in the monetary policy rule can change across regimes according to a state-dependent jump process. In addition, we suppose that the probability of switching toward a more active monetary policy rule is higher when inflation deviates from its targeted level. The three effects we identify are the following:

First, the expectations of future switches affect the economic dynamics differently depending on the size of the shocks. We call this mechanism endogenous expectations formation effect. In our calibration, the probability of a future switch increases with the size of the shock, thus the expectations formation effect is all the larger that a shock is large. As a consequence, in the Fisherian model, real interest rate shocks are less destabilizing than in the state-independent case, especially for large shocks. The standard inflationary effect of such shocks is weakened by the endogenous expectations formation effect, which tends to decrease the immediate response of inflation. When the real interest rate shock is important, the expectations formation effect dominates and leads to a hump-shaped response of inflation. Similarly, in the New-Keynesian model, the state-dependence tends to stabilize output and inflation after a demand shock. Facing a supply shock, this effect tends to destabilize output while it always stabilizes inflation. ${ }^{1}$

Second, shocks and regimes are correlated. In our illustrations, large shocks are, on average, associated with more active monetary policy regime since they induce large inflation deviation from the inflation target. Asymptotically, large shocks are thus more stabilized by an aggressive monetary policy and hence, the implied distribution of inflation is less volatile. We call this mechanism the selection effect.

Third, stemming from these two effects, the state-dependent fluctuations of probabilities undermine the certainty equivalence and lead to distortions in the distributions of endogenous variables. In our examples, larger variance of shocks tends to stabilize the economy as it increases the likelihood of switching toward the active monetary policy regime. Furthermore, large shocks triggers asymmetric distribution. In the Fisherian model, an inflationary shock leads to a left-tailed asymmetric distribution of inflation. Indeed, extra inflationary shocks are associated with higher probability of the active monetary policy regime and hence a more stabilizing policy, while deflationary ones are associated with lower probability. This asymmetry induces a downside inflation risks that does not exist in a situation with no or small shocks.

Related Literature Recent papers have challenged the empirical validity of models based on time-invariant behavior of economic agents. For instance, several papers analyze the

\footnotetext{
${ }^{1}$ This feature comes from the fact that transition probabilities only depend on inflation and not on output
} 
sharp decreases in output and inflation volatility around the mid 80s, the so-called "Great Moderation", by allowing for time-varying economic behavior. Among the competing sources of parameter changes, some papers have allowed for shifts in the parameters of monetary policy rules (Clarida et al., 2000; Lubik and Schorfheide, 2004; Bianchi, 2013), others for breaks in the variance of structural shocks (Sims and Zha, 2006; Justiniano and Primiceri, 2008; Fernández-Villaverde et al., 2010; Liu et al., 2010).

Within the context of forward looking economic agents, the possibility of future regimes switches alters agents' current decision rules (Sims, 1982) through what Leeper and Zha (2003) call the expectations formation effects. In simple calibrated monetary models with Markov switching monetary policy rules, Davig and Leeper (2007) show that these effects can be relatively large and can substantially modify determinacy conditions (see also Farmer et al., 2009a).

In most of the literature, the expectations formation effects are state-invariant as regime switches are exogenous. However, allowing for endogenous regime switching appears useful in many contexts: monetary policy regime (Davig and Leeper, 2008), financial crises (Coe, 2002), defaults (Mendoza and Yue, 2011), political regimes (Rothert, 2009), occasionally binding constraints (Christiano and Fisher, 2000), or sudden stops (Calvo, 1998) among many others. In such contexts, we expect the expectations formation effects to depend on the likelihood of future switches and, hence, on the current state of the economy. Davig and Leeper (2008) show that a monetary policy rule that changes when certain endogenous variables intersect specified thresholds leads to substantial and state-dependent expectations formation effects. However, Davig and Leeper's approach relies on numerical methods that imply high computational costs and lack of analytical results.

Other papers solve non-linear Markov switching rational expectations models invoking perturbation approach without fully justifying the underlying Implicit Function Theorem. Davig and Doh (2008) refer to Woodford (2003) to linearize a non-linear new Keynesian model with Markov switching. Foerster et al. (2011) propose an algorithm to solve a large class of non-linear Markov switching rational expectations models based on successive differentiations. At first sight, these approaches appear more flexible than ours as they allow for solving models with backward-looking components. However, they provide no explicit argument proving that a unique "stable" solution of the original non-linear model exists. In this paper, we fill in this gap by applying adequately the Implicit Function Theorem to Markov switching rational expectations models. More importantly, we innovate by introducing statedependent probability distributions.

This paper is also closely related to the growing literature on solving linear exogenous Markov switching models. We identify two main solution techniques in this literature: the undetermined coefficient approach (Blake and Zampolli, 2006; Davig and Leeper, 2007; Svennson and Williams, 2009) and a direct approach (Farmer et al., 2010b; Cho, 2009). Our approach is closer to the undetermined coefficient approach since both methods are equivalent when models are linear.

The determinacy conditions for bounded solutions are still under debate. Davig and Leeper (2007) propose a simple determinacy condition for forward-looking Markov switching 
models. However, Farmer et al. (2010a) have casted doubts on their methods ${ }^{2}$. Because of this controversy, most of the literature has turned to the Mean Square Stability concept (see Farmer et al., 2009b; Cho, 2009) following the influential book by Costa et al. (2005). However, there is, at this stage, no theoretical argument ensuring the consistency of this concept of stability with the perturbation approach for non linear Markov switching DSGE models ${ }^{3}$.

Disregarding rational expectations, endogenous changes have been well studied in econometric frameworks. Following the seminal paper by Hamilton (1989), Filardo (1994) and Filardo and Gordon (1998) estimate Markov switching regressions with time-varying transition probabilities. More recently, Kim et al. (2003) propose a technique for estimating multivariate models with endogenous regime switching, i.e. where transition probabilities depend on endogenous variables. However, these significant progresses cannot be replicated yet to estimate rational expectations models with endogenous regime switching.

The remainder of the paper is organized as follows. Section 2 presents the class of models we study as well as the concept of solution. Then, we provide our main theoretical result in Section 3, and give its applications in section 4. Finally, we apply our results to a Fisherian model, section 5 and a New-Keynesian model, section 6.

\section{The class of models}

In this section, we present the class of models under consideration and we specify the definition of a stable equilibrium.

Most of recent rational expectations macroeconomic models with regime switching can be reduced to the following system:

$$
\mathbb{E}_{t}\left[f_{s_{t}}\left(z_{t+1}, z_{t}, z_{t-1}, \sigma v_{t}\right)\right]=0
$$

where $z$ is a vector of endogenous variables evolving in a bounded closed set $F$ of $\mathbb{R}^{n}, v$ is a multi-dimensional stochastic process evolving in a bounded domain $V$ of $\mathbb{R}^{p}$ and $\sigma$ is a scalar. We denote by $s_{t}$ in $\{1, \cdots, N\}$ the regime of the economy at date $t$. For any $i, f_{i}$ is a regular function and $\mathbb{E}_{t}$ is the expectation operator given information available at time $t$, namely current and past shocks and regimes.

We study models in which the probability distribution of shocks depends on endogenous variables, $z$. In this case, we abusively say that shocks are endogenous, otherwise they are exogenous. Endogenous shocks are, for instance, shocks with state-dependent variances. In addition, we allow transition probabilities between regimes to depend on endogenous variables also. In this case, we say that the regime switching is endogenous.

More formally, we denote by $u_{t} \in U$ the concatenation of the regime and the shocks

\footnotetext{
${ }^{2}$ In a companion paper (Barthélemy and Marx, 2012), we provide the conditions guaranteing the uniqueness of a bounded solution for linear and forward-looking Markov switching models.

${ }^{3}$ Contrary to standard boundedness concept, Mean Square Stability is not sufficient to ensure that spaces are Banach, whereas this condition is a prerequisite for applying the Implicit Function Theorem
} 
$\left(s_{t}, v_{t}\right)$. The set $U^{\infty}$ represents the set of infinite sequences $u^{t}=\left(u_{t}, u_{t-1}, \cdots\right) .{ }^{4}$ We consider stochastic processes, $u_{t}$, whose conditional probability distribution depends on any policy function, $\phi$, mapping $U^{\infty}$ to $\mathbb{R}^{n}$ and on the history of past shocks and regimes, $u^{t-1} \in U^{\infty}$. We denote this measure by $\mu_{\gamma}$ in $\mathcal{M}(U)$ :

$$
\forall(s, v) \in U, \quad \mu_{\gamma}\left(s, v, \phi, u^{t-1}\right)=h^{s}\left(v, \phi, u^{t-1}\right) p_{\gamma}^{s}\left(\phi, u^{t-1}\right),
$$

where $h^{s}$ is the conditional probability density function of shocks, $u$, in regime $s$ and $p_{\gamma}^{s}$ the (unconditional) probability of regime $s$. The scale parameter $\gamma$ measures the sensitivity of the probability to the policy function $\phi$. When $\gamma=0, p^{0}$ is independent from $\phi$ and we say that the regime switching is exogenous. We provide some useful properties of the class of measure $\mu_{\gamma}$ in Appendix A.1 under smoothness conditions on $h^{s}$ and $p_{\gamma}^{s}$.

The standard perturbation approach for models without regime switching (Woodford, 1986; Jin and Judd, 2002, see) consists of considering a continuum of models with different sizes of shocks indexed by $\sigma$. When $\sigma=0$, the shocks do not affect the economic model. Then we link he determinacy of the stochastic model with the deterministic one (the one with no shock) by applying the Implicit Function Theorem (IFT).

In this paper, we innovate by considering a continuum of measures, $\mu_{\gamma}$, to solve the endogenous regime switching model. When $\gamma=0$, the model is an exogenous regime switching rational expectations model. $\gamma$ thus measures the degree of endogeneity of regime switching. We show in section 3 that the determinacy of the endogenous regime switching, when $\gamma$ is small enough, follows from the determinacy of the exogenous regime switching model. The following definition introduces the solution space we consider here.

Definition 1. A stationary rational expectations equilibrium (s.r.e.e.) of model (1) is a continuous function $\phi: U^{\infty} \rightarrow F$ such that:

1. $\|\phi\|_{\infty}=\sup _{U^{\infty}}\left\|\phi\left(u^{t}\right)\right\|<\infty$.

2. If $u$ is a $U$ valued stochastic process whose conditional probability distribution is $\mu_{\gamma}$ (see Equation 2), then $z_{t}=\phi\left(u^{t}\right)$ is a solution of Equation (1).

We restrict our analysis to continuous and bounded functions, and we denote by $\mathcal{B}$, the set of such functions. ${ }^{5}$ This definition is more restrictive than mean-square stability as required by Farmer et al. (2007). However, this stability concept is not suitable for applying a perturbation approach because it does not rely on a norm. On the contrary, the boundedness stability defined above is consistent with a norm defining a Banach space. In addition, our solution space is very close to, but slightly tighter than, the set of essentially bounded functions considered by Woodford (1986). We cannot follow the Woodford's exact definition since the concept of essentially boundedness relies on an intrinsic measure, which does not exist in our setup.

\footnotetext{
${ }^{4}$ For more details about this formalism, see Woodford (1986).

${ }^{5}$ Precisely, $\mathcal{B}$ is the set of functions $\phi$ on $U^{\infty}$ such that, for all $s$, the map $v \mapsto \phi(s, v)$ is continuous and such that $(s, v) \mapsto \phi(s, v)$ is bounded.
} 
We will prove the existence and the uniqueness of a s.r.e.e. when the continuous shocks are small enough (small $\sigma$ ) and the regime switching is weakly endogenous (small $\gamma$ ) by applying the IFT to an operator acting on the Banach space of bounded and continuous functions, $\mathcal{B}$.

\section{Perturbation approach}

The aim of this section is to prove that there exists a unique s.r.e.e. of the model with small continuous shocks ( $\sigma$ small enough) and weakly endogenous regime switching ( $\gamma$ small enough) if the underlying exogenous regime switching model admits a unique solution [Theorem 1].

We prove this result by applying the IFT in Banach Spaces (see Abraham et al., 1988, and section B.1 in the Appendix). The problem can indeed be rewritten as finding the zeros of an operator $\mathcal{N}$ acting on a bounded function $\phi$ in $\mathcal{B}$, the two scale parameters $\gamma$ and $\sigma$ with values in $\mathcal{B}$, such that, for any $u^{t}$ :

$$
\mathcal{N}(\phi, \gamma, \sigma)\left(u^{t}\right)=\int_{U} f_{s_{t}}\left(\phi\left(u u^{t}\right), \phi\left(u^{t}\right), \phi\left(u^{t-1}\right), \sigma v_{t}\right) \mu_{\gamma}\left(u, \phi, u^{t}\right) d u
$$

As explained in section 2, we assume that the transition probabilities do not depend on $\phi$ when $\gamma$ is equal to 0 , and that there is no continuous shock when $\sigma=0$. Thus for any regime and shocks, $u^{t} \in U^{\infty}$ :

$$
\mathcal{N}(\phi, 0,0)\left(u^{t}\right)=\sum_{i=1}^{N} p_{0}^{i}\left(u^{t}\right) \int_{V} f_{s_{t}}\left(\phi\left(i s^{t}, v v^{t}\right), \phi\left(u^{t}\right), \phi\left(u^{t-1}\right), 0\right) h^{i}\left(v, \phi, u^{t-1}\right) d v
$$

This operator corresponds to a model with exogenous regime switching and the shocks, $v$, are sunspot shocks (do not appear in the model, $f_{s_{t}}$ ). One may notice that transition probabilities can depend on past regimes and shocks. This thus encompasses Markov Switching models. Besides, even if the shocks, $v$, are sunspots, their probability distribution functions can depend on the policy function, $\phi$.

We introduce $\mathbb{E}_{t}^{0}$ the expectation operator corresponding to exogenous regime switching and potentially endogenous continuous shocks. Equation (4) can then be rewritten as:

$$
\mathcal{N}(\phi, 0,0)\left(u^{t}\right)=\mathbb{E}_{t}^{0} f_{s_{t}}\left(\phi\left(u^{t+1}\right), \phi\left(u^{t}\right), \phi\left(u^{t-1}\right), 0\right)
$$

We will apply the IFT to $\mathcal{N}$ to prove the existence of a unique s.r.e.e. In order to do that, it is necessary to know a particular solution when $\gamma=0$, and $\sigma=0, \phi_{0}$, and to check for the invertibility of the differential, $D_{\phi} \mathcal{N}\left(\phi_{0}, 0,0\right)$.

In fact, the invertibility of $D_{\phi} \mathcal{N}\left(\phi_{0}, 0,0\right)$ coincides with the existence of a unique bounded continuous function, $h$, satisfying for any bounded continuous function, $\psi \in \mathcal{B}$ :

$$
D_{\phi} \mathcal{N}\left(\phi_{0}, 0,0\right)(h)=\psi
$$


The invertibility of $D_{\phi} \mathcal{N}\left(\phi_{0}, 0,0\right)$ corresponds to the existence of a unique s.r.e.e, for any bounded stochastic process, $\psi_{t}$, of the following "linearized" regime switching model (see Appendix B.1):

$$
\mathbb{E}_{t}^{0}\left[A\left(s^{t+1}\right) z_{t+1}\right]+B\left(s^{t}\right) z_{t}+C\left(s^{t}\right) z_{t-1}=\psi_{t},
$$

where the shocks $u_{t}$ follow the probability distribution $\mu_{0}$, i.e. the regime switches are exogenous while the shocks may follow state dependent probability distributions. The matrices $A\left(s^{t+1}\right), B\left(s^{t}\right)$ and $C\left(s^{t}\right)$ depend on the derivatives of $f_{s_{t}}$ and on the regimes history. The coincidence between the invertibility of $D_{\phi} \mathcal{N}\left(\phi_{0}, 0,0\right)$ and the uniqueness of a solution of model (6) leads to the following theorem.

Theorem 1. If there exists a continuous function $\phi_{0}:\{1, \cdots, N\}^{\infty} \rightarrow F$ such that

1. $\phi_{0}$ is a particular s.r.e.e. of the non-linear exogenous regime switching model without shocks $\left(\mathcal{N}\left(\phi_{0}, 0,0\right)=0\right)$.

2. The linearized model 6 admits a unique s.r.e.e. for any bounded process $\psi_{t}$.

Then there exists $\gamma_{0}$ and $\sigma_{0}$ small enough such that, for any $\gamma$ smaller than $\gamma_{0}$, any $\sigma$ smaller than $\sigma_{0}$, there exists a unique s.r.e.e. of model 3 around $\phi_{0}$. Furthermore, the smoothness of $\phi$ stems from the one of the model, and its expansion in $\gamma$ and $\sigma$ is given by the differentiation of the implicit relation:

$$
\mathcal{N}(\phi(\gamma, \sigma), \gamma, \sigma)=0
$$

Proof. This theorem is a direct application of IFT in Banach Spaces. See Appendix B.1 for more details.

The first hypothesis of theorem 1 can usually be checked by hand while the second hypothesis coincides with the determinacy conditions of the underlying linearized model. Contrary to Woodford (1986), this theorem only offers sufficient conditions of determinacy but is silent on the reciprocal. In addition, the solution, $\phi(\gamma)$, is not necessarily recursive contrary to what is postulated by Jin and Judd (2002).

In most cases, these conditions are however difficult to verify as the coefficients of model 6 may depend on all the history of past regimes. Section 4 gives examples for which we can explicitly derive condition 2 . of Theorem 1 .

\section{Applying Theorem 1}

In this section, we apply Theorem 1 to two disconnected cases: (i) when there is no regime switching and shocks are endogenous (ii) when shocks are exogenous but regimes switch endogenously. More general results can be obtained by mixing these two cases, but we prefer splitting them for the sake of simplicity. 


\subsection{Endogenous shocks, no regime switching}

The first corollary of Theorem 1 gives a rationale for linearizing a rational expectation model with endogenous shocks, when they are small enough. To this aim, we consider the model 3 in the absence of regime switching:

$$
\mathbb{E}_{t}\left[f\left(z_{t+1}, z_{t}, z_{t-1}, \sigma v_{t}\right)\right]=0
$$

where $v^{t}$ follows a continuous law, $h\left(v, \phi, v^{t-1}\right)\left(\mu_{\gamma}=h\right)$. We assume that $h$ is Lebesguecontinuous and $C^{1}$ according to its second component.

We also suppose that the non linear model 8 admits a constant solution when $\sigma=0$. We denote it by $\bar{z}$ and call it the deterministic steady-state. Thus, $\bar{z}$ satisfies $f(\bar{z}, \bar{z}, \bar{z}, 0)=0$. Theorem 1 leads to the following proposition.

Proposition 1. The linearized model of the non linear model 8 at $\bar{z}$ is given by:

$$
\partial_{1} f(\bar{z}, \bar{z}, \bar{z}, 0) \mathbb{E}_{t}^{0} z_{t+1}+\partial_{2} f(\bar{z}, \bar{z}, \bar{z}, 0) z_{t}+\partial_{3} f(\bar{z}, \bar{z}, \bar{z}, 0) z_{t-1}+\sigma \partial_{4} f(\bar{z}, \bar{z}, \bar{z}, 0) v_{t}=0,
$$

where $v_{t}$ follows a continuous exogenous process, $v_{t} \sim h\left(\cdot, \bar{z}, v_{t-1}\right)$. If the linearized model 9 is determinate, then for $\sigma$ small enough, model (8) admits also a unique s.r.e.e.

Proof. The linearized model 9 is a well-studied linear rational expectations model with $e x$ ogenous shocks (see Appendix B.2 for more details), and determinacy conditions have been established by Blanchard and Kahn $(1980)^{6}$.

This result generalizes Theorem 2 by Woodford (1986) to the case of shocks with statedependent probability distributions. Determinacy conditions of the non-linear model with endogenous shocks are unaffected by the fluctuations of probability distribution insofar as shocks are small. The standard linearization taken at the constant probability distribution gives the determinacy condition of the endogenous shocks model. When unique, the firstorder expansion of the solution exactly coincides with the solution of the linearized model (Equation 9). The endogeneity of shocks' probability thus only matters for the second and higher orders Taylor expansion of the solution. We give the details in Appendix B.2.

\subsection{Exogenous Shocks, Endogenous Regime Switching}

Let us now turn to the endogenous regime switching model with exogenous shocks. We consider the following purely forward-looking model:

$$
\mathbb{E}_{t}\left[f_{s_{t}}\left(z_{t+1}, z_{t}, \sigma v_{t}\right)\right]=0 \quad \text { with } \quad v_{t+1}=\Lambda v_{t}+\nu_{t+1},
$$

where $\nu_{t}$ is a centered standardized truncated Gaussian. We assume that the transition probability from regime $i$ to regime $j$ only depends on the past value of endogenous variables,

\footnotetext{
${ }^{6}$ Klein (2000) extends Blanchard and Kahn (1980) when the matrix $\partial_{1} f(\bar{z}, \bar{z}, \bar{z}, 0)$ is singular.
} 
$\phi\left(u^{t-1}\right)$. Thus, there exists a function $p_{i j}$ with values in $[0,1]$ such that:

$$
p_{\gamma}^{j}\left(\phi,\left(i s^{t-1}, v^{t}\right)\right)=p_{i j}\left(\gamma, \phi\left(s^{t-1}, v^{t-1}\right)\right) .
$$

In addition, we assume that the probabilities $p_{i j}$ are smooth $\left(C^{1}\right)$ and constant for $\gamma=0$ $\left(p_{i j}(0,)=.\bar{p}_{i j}\right)$. The implied measure, $\mu_{\gamma}$, belongs to the class of measures described in Section 2 .

As in the absence of regime switching, we assume that there exists a solution, $\phi_{0}$, when there is no shock. In addition, we suppose that this solution only depends on the current regime, i.e., $\phi_{0}\left(i s^{t-1}\right)=\bar{z}_{i}$ where $\left(\bar{z}_{1}, \cdots, \bar{z}_{N}\right)$ is solution of the following system of $N \times n$ equations:

$$
\forall i \in\{1, \cdots, N\}, \quad \sum_{j=1}^{N} \bar{p}_{i j} f_{i}\left(\bar{z}_{j}, \bar{z}_{i}, 0\right)=0
$$

In this context, the associated linearized model can be written as:

$$
\mathbb{E}_{t}^{0}\left[A_{s_{t} s_{t+1}} x_{t+1}\right]+B_{s_{t}} x_{t}=\psi_{t}
$$

where $A_{i j}$ and $B_{i}$ depend on the first derivatives of $f_{i}$ in $\left(\bar{z}_{j}, \bar{z}_{i}, 0\right)$ and are explicitly defined in Equation (32) in Appendix C.1. $\mathbb{E}_{t}^{0}$ denotes the expectation operator with respect to the state-invariant measure, $\mu_{0} . \psi_{t}$ is any bounded stochastic process. Finally, we impose that $B_{i}$ is invertible for any $i$.

This class of linear Markov switching models has been studied by Cho (2009) and Farmer et al. (2009b) using mean square stability and by Barthélemy and Marx (2012) using boundedness stability. A refinement of Barthélemy and Marx (2012) leads to the following determinacy condition:

Proposition 2. The linearized model 13 admits a unique s.r.e.e if and only if the limit of the sequence $u_{p}=\left(\sum_{\left(k_{1}, \cdots, k_{p-1}, k_{p}\right) \in\{1, \cdots, N\}^{p}} \bar{p}_{i k_{1}} \cdots \bar{p}_{k_{p-1} j}|| \mid B_{k_{1}}^{-1} A_{k_{1} k_{2}} \cdots B_{k_{p-1}}^{-1} A_{k_{p-1} k_{p}} \|\right)^{1 / p}$ is smaller than 1.

Proof. For the details on the proof, we refer to Barthélemy and Marx (2012) and Appendix C.1.

This condition extends the standard Blanchard and Kahn's forward iteration in a context of regime switching. In this context, not only the transition matrices, $B_{s_{t}}^{-1} A_{s_{t}}$, should be contracting for any regime but also all possible products between these matrices. Hence, the determinacy condition depends on the limit of a sequence that can be cumbersome to compute. Therefore, the determinacy conditions can be difficult to check in practice. Barthélemy and Marx (2012) give ways to circumvent this computational difficulty. We refer to this paper for further details.

Proposition 2 combined with Theorem 1 leads to the following result. 
Proposition 3. Under conditions of Proposition 2, a unique s.r.e.e of model (10) exists for small shocks and weakly endogenous transition probabilities ( $\sigma$ and $\gamma$ small enough). In this case, the first-order expansion of the solution is given by:

$$
\phi\left(v^{t}, s^{t}, \sigma, \gamma\right)=\bar{z}_{s_{t}}+\gamma r_{s_{t}}+\sigma R_{s_{t}} v_{t}+o(\sigma, \gamma),
$$

where $R_{s_{t}}$ and $r_{s_{t}}$ are given in Appendix C.2, equations (35) and (36).

In addition, if the steady-state $\phi_{0}$ does not depend on the current regime, $\left(\bar{z}_{s_{t}}=\bar{z}\right)$, the second-order expansion of the solution is as follows:

$$
\phi\left(v^{t}, s^{t}, \sigma, \gamma\right)=\bar{z}+\sigma R_{s_{t}} v_{t}+\sigma^{2} q\left(s_{t}, v_{t}\right)+\sigma \gamma h_{s_{t}} v_{t}+o\left((\sigma, \gamma)^{2}\right),
$$

where $h_{s_{t}}$ and $q$ are given in Appendix C.2, (37), (38). Moreover, the coefficients of $\gamma^{k}$ are zero for any order $k>0$.

This proposition calls for many comments.

First, when the steady-state differs across regimes, state-dependent transition probabilities affect the endogenous variables in each regime even in the absence of shocks. The first two left-hand-side terms of Equation (14) provide the first-order approximation of the equilibrium in the absence of shocks with respect to $\gamma$. Higher-order terms in $\gamma^{k}$ correct for higher moments of the transition probabilities.

Second, the first-order expansion of the solution with respect to the shocks coincides with the one when transition probabilities are constant (Equation 14). Thus, the state-dependence of probabilities only alters the impact of shocks at higher orders.

Third, when the steady-state is regime invariant, the endogeneity of transition probabilities only modifies the transmission of shocks but does not modify the equilibrium in the absence of shocks -all the terms in $\gamma^{k}$ are zero.

Fourth, when the steady state is regime invariant, and the shocks are uncorrelated, endogeneity does not matter. For i.i.d. shocks, the expectations of future shocks are constant and, hence, do not modify the equilibrium independently from the probabilities of regime switches. In this case, there is no expectation formation effect, i.e. the expectations of future shocks do not affect the current decision rule.

When a forward-looking regime switching model is linear, the solution is defined whatever the size of the shocks. Therefore, there is no necessity to assume small shocks. We thus present a refinement of proposition 3 in the case of linear model - $f_{i}$ is linear for any $i \in$ $\{1, \cdots N\}$ ), with a constant steady-state. In this specific case, Equation (10) appears to be very similar to a linear Markov switching model apart from the underlying transition probabilities:

$$
A_{s_{t}} \mathbb{E}_{t}\left(z_{t+1}\right)+B_{s_{t}} z_{t}+C_{s_{t}} v_{t}=0 .
$$

The linearized version of this Equation is thus a linear Markov switching model with constant transition probabilities. If we denote by $\mathbb{E}_{t}^{0}$ the conditional expectations given constant transition probabilities, the linearized model is: 


$$
A_{s_{t}} \mathbb{E}_{t}^{0}\left(z_{t+1}\right)+B_{s_{t}} z_{t}=\psi_{t}
$$

where $\psi_{t}$ is any bounded stochastic process.

The following proposition replicates proposition 3 in the specific case of linear models and shows that any order of the solution solves an exogenous regime switching model.

Proposition 4. If the condition of Proposition 2 is satisfied, the linear regime switching rational expectation model 16 admits a unique s.r.e.e., $z_{t}$, for $\gamma$ small enough. In addition, if the probabilities are polynomial, the solution follows, for any integer $K$ :

$$
z_{t}=\sum_{k=0}^{K} \gamma^{k} w_{t}^{k}+o\left(\gamma^{K}\right)
$$

where the sequence $w_{t}^{k}$ depends only on current shocks $\left(s_{t}, v_{t}\right)$ and is defined recursively as the solution of the following exogenous regime switching model:

$$
A_{s_{t}} \mathbb{E}_{t}^{0}\left(w_{t+1}^{k}\right)+B_{s_{t}} w_{t}^{k}=P_{k, s_{t}}\left(v_{t}\right),
$$

where $P_{k, s_{t}}$ is recursively defined as a polynomial of $v_{t}$ depending on $\left\{w_{j}\right\}_{j<k}$ (see Appendix $D$ equation (40) for the exact definition of $P_{k, s_{t}}$ ). The two first functions are defined by:

$$
\begin{gathered}
P_{0, s_{t}}=-C_{s_{t}} v_{t} \\
P_{1, s_{t}}=-\gamma \sum_{j} \partial_{1} p_{s_{t} j}\left(0, w_{t}^{0}\right) \mathbb{E}_{t}^{0}\left[w_{t+1}^{0} \mid s_{t+1}=j\right] .
\end{gathered}
$$

Proof. This Proposition follows from Proposition 2. Proof is given in Appendix D.

This proposition shows that solving an endogenous regime switching model only requires solving multiple exogenous regime switching models. The zero order expansion is exactly the solution of the regime switching model with constant probabilities. The first-order expansion solves the same model but instead of the shocks, $-C_{s_{t}} v_{t}$, the right-hand-side member corresponds to the first-order wedge introduced by the state-dependence of transition probabilities. This term is the (first-order) additional expectations formation effect caused by expected fluctuations of transition probabilities.

This proposition is tractable as, at any order, the coefficient $w^{k}$ in the Taylor expansion is solution of an exogenous regime switching model with the right-hand-side only depending on lower order terms, $\left\{w_{j}\right\}_{j<k}$. Applying proposition 4 thus requires two steps: first, at each order, we need to compute $w_{k}$ given past $w_{j}$; second, we have to solve a regime switching model with polynomial or analytic function of shocks, $v_{t}$. We describe in Appendix D an algorithm to compute the Taylor expansion in $\sigma$ at any order. 


\section{A Fisherian model of inflation determination}

In this section, we apply our results to a Fisherian model of inflation determination with two monetary policy regimes that switch endogenously. We consider a Fisherian equation linking the nominal and the ex-ante real interest rate. In addition, monetary authority sets the nominal interest rate by following an interest rate rule. Its reaction to inflation may change across monetary policy regimes. When the transition probabilities are constant, Davig and Leeper (2007) have established determinacy conditions for this model - called the Long Run Taylor Principle - saying that a combination between the transition probabilities and the central bank's reaction to inflation parameters has to be greater than one. Applying Proposition 4, we obtain the equilibrium dynamics when transition probabilities depend on inflation.

We identify two mechanisms stemming from the endogeneity of transition probabilities. First, the expectations formation effects due to the possibility of future switches are state contingent, whereas these effects are constant in an exogenous Markov switching model. Second, the endogenous fluctuations of transition probabilities lead to a state-dependent likelihood of each regime after a shock. This selection effect triggers modifications of averaged Impulse Response Functions compared to those obtained for exogenous regime switching. When the probability of having a more reactive central banker increases with inflation, these two effects tend to stabilize the economy and especially in the presence of large shocks. These effects finally modify the shape of inflation distributions qualitatively.

\subsection{The model}

Consider a nominal bond that costs 1 at date $t$ and pays off $1+i_{t}$ at date $t+1$. The asset pricing equation for this bond can be written in $\log$ form around a constant steady state as:

$$
i_{t}=\mathbb{E}_{t}\left(\pi_{t+1}\right)+r_{t},
$$

where $r_{t}$ is the ex-ante equilibrium interest rate and evolves as

$$
r_{t}=\rho r_{t-1}+v_{t}
$$

where $\rho<1$ and $v_{t}$ is a zero-mean i.i.d bounded process. Monetary policy follows a simplified Taylor rule, adjusting the nominal interest rate in response to inflation, where the reaction to inflation evolves stochastically across regimes,

$$
i_{t}=\alpha_{s_{t}} \pi_{t},
$$

where $s_{t}$ is the realized (observable) monetary policy regime and takes two values $\{1,2\}$. We use the formalism introduced in section 4.2 and assume that the switching process follows a Markov chain with transition probabilities $p_{i j}=p\left(s_{t}=j \mid s_{t-1}=i\right)$ depending on past 
inflation, $\pi_{t-1}$. To simplify the exposition, we focus on quadratic probabilities satisfying:

$$
p_{i j}\left(\pi_{t-1}\right)=\bar{p}_{i j}+\gamma \lambda_{i j} \pi_{t-1}^{2},
$$

where $\lambda_{i j}$ is a parameter reflecting the sensitivity of the probability, $p_{i j}$, to inflation and $\gamma$ is the scale parameter. For consistency, $\sum_{i} \bar{p}_{i j}=1$ and $\sum_{i} \lambda_{i j}=0$. Furthermore, we assume that $\gamma$ is small enough to guarantee that the probabilities remain between 0 and 1 .

As mentioned by Filardo (1994), endogenous regime switching as exemplified by equation (22) allows for state-dependent duration of each regime. In our example, if $\lambda_{11}$ is negative, the average duration of regime 1 decreases when inflation deviates from its steady-state.

\subsection{The solution}

We assume that $\left|\alpha_{1}\right|>\bar{p}_{11}$ and $\left|\alpha_{2}\right|>\bar{p}_{22}$ and we apply Proposition 4 to find determinacy conditions of the Fisherian model and the first-order Taylor expansion of the solution.

Proposition 5. If the policy parameters satisfy the following determinacy condition:

$$
\left|\alpha_{1}\right| \cdot\left|\alpha_{2}\right|+\bar{p}_{22}\left(1-\left|\alpha_{1}\right|\right)+\bar{p}_{11}\left(1-\left|\alpha_{2}\right|\right)>1 .
$$

Then there exists a unique s.r.e.e. for $\gamma$ small enough and the solution satisfies:

$$
\pi_{t}=-\frac{r_{t} \Lambda_{s_{t}}}{\alpha_{s_{t}}}+\rho \gamma\left[c_{s_{t}} r_{t}^{3}+d_{s_{t}} \frac{\operatorname{var}(v)}{1-\rho^{2}} r_{t}\right]+o(\gamma),
$$

where $c_{s_{t}}, d_{s_{t}}$ and $\Lambda_{s_{t}}$ are regime-dependent scalars (see Appendix $E$ for their expressions)

Proof. This Proposition is an application of Propositions 2 and 4. See appendix E for the complete proof.

As emphasized by Theorem 1, the determinacy conditions coincide with those of the exogenous Markov switching model. Thus, condition (23) is similar to determinacy conditions by Farmer et al. (2009a) and extends those obtained by Davig and Leeper (2007). The interpretation of equation (23) is similar to the long-run Taylor principle by Davig and Leeper (2007): there may exist a unique s.r.e.e. even if policy deviates from the Taylor principle "substantially for brief periods or modestly for prolonged periods".

Equation (24) gives the first-order Taylor expansion of inflation with respect to the scale parameter. The first term exactly coincides with the solution of the model when probabilities are constant. The second term results from the expectations of the third raw moments of the real interest rate shock. Generically, the higher the variance of the real interest rate shock, the more the endogeneity of probabilities matters.

Let us consider a central bank that can switch between an active monetary policy regime $\left(\alpha_{2}=3\right)$ and a passive monetary regime in which the monetary authority responds less than one to one to inflation $\left(\alpha_{1}=0.9\right)$. Furthermore, we assume that the higher the deviation from steady-state inflation, the more likely the central bank chooses the active regime $\left(\lambda_{11}<0\right)$. 
The parameters are fixed to deliver plausible Impulse Response Functions (see Table 1 in Appendix G). In this numerical application, a deviation of $1 \%$ from the steady-state level of inflation decreases the probability of remaining in regime 1 by $-0.4 \%$. In this case, the first-order Taylor expansion of inflation (in $\gamma$ ) is:

$$
\begin{gathered}
\text { When } s_{t}=1, \quad \tilde{\pi}_{t}=(6.15-0.15 \gamma) \tilde{r}_{t}-75 * 10^{2} \gamma \tilde{r}_{t}^{3} . \\
\text { When } s_{t}=2, \quad \tilde{\pi}_{t}=(0.60-0.004 \gamma) \tilde{r}_{t}-1.4 * 10^{2} \gamma \tilde{r}_{t}^{3} .
\end{gathered}
$$

As we could expect, the responses to a real interest rate shock in both regimes are smaller than in the exogenous regime-switching $(\gamma=0)$ since the probability of switching toward the aggressive regime increasingly anchors expectations. In what follows, we discuss two new features of the state-dependent regime switching equilibrium in comparison with the standard exogenous Markov switching model as studied in Davig and Leeper (2007): the endogenous expectations formation effects and the endogenous selection effect.

\subsection{Endogenous Expectations Formation Effects}

In this section, we analyze the impact of the state-dependence of transition probabilities on expectations. In exogenous Markov switching models, Leeper and Zha (2003) and Davig and Leeper (2007) show that the possibility of future switches alters the current decisions of economic agents following the suggestion by Sims (1982). This mechanism is known as the Expectations Formation Effect. Higher probabilities of switching from one regime to another amplify these effects. Therefore, one may expect that these effects become state-dependent in an endogenous regime switching framework.

To only focus on these endogenous expectations formation effects, we study the response of inflation to a real interest rate shock in each regime -i.e. when the economy remains unexpectedly in the same regime and no new shock hit the economy after the initial shock. We plot these functions in figure 1. The left graph depicts the response to a one-standard deviation shock while the right graph displays the inflation response to a ten-standard deviation shock. The blue (red resp.) line represents the response when monetary policy is always active (passive resp.). The dashed thin line displays the response of $r_{t}$ to a real interest shock $v_{t}$. We gather all the responses for different sizes of the shock in figure 2 . In the left graph, in blue (in the right graph, in red resp.), we represent the ratio between the contemporaneous response to a shock and the size of the shock in the active (passive resp.) regime. The dashed thick line represents the maximal response divided by the size of the shock in each regime. Finally, in dashed thin line, we plot these multipliers when probabilities are constant $(\gamma=0)$. From figures 1 and 2, we draw four main conclusions.

First, whatever the size of the shock, the multipliers in both regime are strictly lower than those of the corresponding exogenous Markov switching model (see figure 2). This means that the threat of switching toward a more aggressive regime when the inflation is higher helps stabilizing the economy in both regimes.

Second, there is a wedge between the exogenous solution and the endogenous one due to 
the uncertainty around the future values of shocks. This wedge is illustrated by the little difference between the dashed line and the red line when shocks are close to zero and by the variance term in equation (24).

Third, the larger the shock (in absolute value), the lower the multiplier. In the exogenous case, the size of the shock proportionally shifts the response of inflation as the solution is linear (the multiplier is constant, see figure 2). In the endogenous regime switching, a larger shock leads to a relatively smaller response of inflation. The initial response to a shock divided by the size of the shock is quadratic in the size of the shock (figure 2). We notice that the symmetric shape is a consequence of the quadratic term ${ }^{7}$ in (22), and stems from the cubic term in equation (24). In other words, the impact of the expectations is higher when the shock is larger. Indeed, a larger shock triggers larger expectations of future switches toward the active monetary policy regime, and this tends to stabilize expectations. This endogenous expectations formation effect can be so important that it also alters the inflation responses in the active monetary policy regime itself. In such a situation, even if the probability of switching to the passive regime has not changed, economic agents internalize that if the economy switches to the passive monetary regime, the probability of staying in this regime has decreased. Consequently, the multiplier in the active regime also depends on the size of the shocks.

Fourth, when the size of the shock is very large, it generates hump-shaped inflation response (see figure 1). Actually, the cubic polynomial (25) admits a maximum, $r_{M}$. If $r_{t}>r_{M}$, the response to the shock first increases then decreases, leading to a hump-shape.

The causes of the hump-shape and of the decreasing multiplier are the combination of two mechanisms. First, in an exogenous regime switching, a positive shock on the real interest rate, $r_{t}$, leads to a rise in inflation. In the passive regime, this rise increases the probability of switching to the more active monetary policy regime, and through the expectations formation effect tends to limit inflationary pressures. While the first impact is linear, the second is not. For small shocks, the first mechanism is preeminent and explains the monotonic decrease of inflation in reaction to shocks. The larger the shock, the more important the second mechanism. When this mechanism becomes predominant, the initial reaction of inflation is toned down by the threat of switching to the active regime. The mechanic decrease of the shock due to autocorrelation reduces this second channel and hence increases inflation. At a certain threshold, the two mechanisms cancel out, explaining the hump-shape.

To sum up, endogenous regime switches trigger endogenous expectations formation effects which are absent in exogenous regime switching models. This new mechanism seems particularly desirable in a large number of economic issues such as occasionally binding constraints, zero lower bound or defaults.

\subsection{Endogenous Selection Effect}

We now turn to the direct implication of the state-dependence of transition probabilities. We give the name of selection effect to the difference between the impact of a shock when agents

\footnotetext{
${ }^{7}$ Assuming linear probabilities leads to an asymmetric behavior
} 
expect that transition probabilities are state-dependent but regime switches exogenously and the impact when regime switches endogenously and agents rationally takes into account this endogeneity. While the endogenous expectations formation effects modifies the contemporaneous response to a shock, the selection effect is only visible if we observe multiple trajectories as it modifies the occurrence of each regime. The endogenous expectations formation effect stems from the selection effect. In the Fisherian model, the selection effect reflects the higher likelihood of having an active central banker after large shocks compared to the case in which transition probabilities were constant.

We represent in figure 3 the expected impulse response function to a real interest rate shock (in red) for small and large shocks assuming that the initial regime is the passive one. We also plot the expected response (in blue) considering a model in which inflation can switch between the two values given by equations (25) and (26) according to a Markov process with constant transition probabilities $\left(\bar{p}_{11}\right.$ and $\left.\bar{p}_{22}\right)$. The difference between the two curves is only a consequence of a selection effect due to endogenous fluctuations of transition probabilities.

When shocks are small, the selection effect is negligible (figure 3). The larger the shock, the larger the selection effect. This finding is consistent with the fact that transition probabilities are quadratic and hence more sensitive to large inflation deviations and thus to large shocks. After a large shock, inflation increases in both regimes. The likelihood of the active monetary policy regime increases. Thus, the average inflation response is closer to the one under the active monetary policy regime.

Finally, in our application, we emphasize a direct implication of the state-dependence on the likelihood of each regime; this leads to complex behavior of expected IRFs through what we call the selection effect. This mechanism is already observed in endogenous regime switching model without rational expectations.

\subsection{Consequences on inflation distributions}

The two effects mentioned above, endogenous expectations formation effect and selection effect, contribute to modify significantly the shape of the distribution probability functions of inflation. The variance as well as the skewness change with respect to the size of the shocks.

Figure 4 plots the distribution of inflation after a 10-standard deviation real interest rate shock when the initial monetary regime is passive and shocks hit the economy stochastically. The left, middle and right panels report the distributions of inflation two, ten and forty quarters after the shock hits the economy. The dashed black curve displays the distribution for the exogenous regime switching model $(\lambda=0)$. The blue curve displays the distribution when economic agents internalize that probabilities are state-dependent but regime switching probability are actually constant. The red curve displays the distribution of inflation when probabilities are state-dependent and agents. The difference between the dashed and the blue curves results from the expectations formation effects only, while the endogenous selection effect triggers the difference between the blue and the red curves. These assessments of the respective role of the two effects on the distributions are only qualitative since the model is 
calibrated and very simple.

Immediately after a large real interest shock, the expectations formation effect dominates. Even if the shock increases the probability of switching toward a more active monetary policy regime, the economy takes time to switch explaining why the selection effects is second order just after the shock. The expectations formation effect generates three main effects. First, average inflation is stabilized consistently to figure 1. Second, volatility is reduced as large shocks are more stabilized when transition probabilities are state-dependent. Third, the expectations of future switches tend to stabilize more large positive shocks rather than negative ones as the level of inflation is already high. A positive shock in addition to the initial large shock tends to increase the probability of switching toward a more active monetary policy regime, while a negative shock decreases this probability. These forces lead to an asymmetric left-tailed distribution.

Longer after the shock, the selection effect matter more. The selection effect asymptotically dominate the expectations formation effect. Both effects contribute to a tighter ergodic distribution than that caused by the exogenous regime switching model. Indeed, after a number of periods the correlation between the size of the shocks and the regime generates a less volatile inflation distribution.

\section{A New-Keynesian model}

The Fisherian model allows understanding certain effects caused by endogenous regime switching in a simple and tractable framework. We delve further into these effects in a more realistic model and solve a standard regime switching new-Keynesian model as suggested by Lubik and Schorfheide (2004) and first studied in a regime switching context in Davig and Leeper (2007). We consider a canonical new-Keynesian model in which the monetary policy rule differs across regimes. As in the previous section, we assume that transition probabilities only depend on inflation.

$$
\begin{gathered}
y_{t}=\mathbb{E}_{t} y_{t+1}-\sigma\left(i_{t}-\mathbb{E}_{t} \pi_{t+1}\right)+\epsilon_{t}^{d} \\
\pi_{t}=\beta \mathbb{E}_{t} \pi_{t+1}+\kappa y_{t}+\epsilon_{t}^{s} \\
i_{t}=\alpha_{s_{t}} \pi_{t}+\gamma_{s_{t}} y_{t}
\end{gathered}
$$

where $y_{t}, \pi_{t}$ and $i_{t}$ are the output-gap, inflation (in $\log$ ) and the nominal interest rate (in deviation around a certain steady state). Equation (27) is an IS curve linking the output-gap to all the future ex-ante real interest rates and future and current demand shocks, $\epsilon_{t}^{d} . \sigma$ is agents' risk aversion. Equation (28) is a New-Keynesian Philips Curve linking inflation to all the future marginal costs summarized by the output-gap where $\kappa$ measures the degree of nominal rigidities while $\beta$ stands for the discount factor. $\epsilon_{t}^{s}$ denotes a cost-push shock translating the Philips Curve. Equation (29) is a simplified Taylor rule with a potential shift in the reaction to inflation $\left(\alpha_{1}\right.$ or $\left.\alpha_{2}\right)$ and output gap $\left(\gamma_{1}\right.$ and $\left.\gamma_{2}\right)$. $s_{t} \in\{1,2\}$ stands for the current regime of monetary policy. Finally, we assume that shocks are AR(1) with a 
persistence equals to $\rho$.

For an extended discussion about the determinacy conditions of this model we refer to Barthélemy and Marx (2012). We fix parameter values as in Lubik and Schorfheide (2004) and Davig and Leeper (2007). Regime 1 is supposed to correspond to the active monetary policy undertaken during the post-Volcker period $\left(\alpha_{1}=2.19\right.$ and $\left.\gamma_{1}=0.3\right)$, while regime 2 stands for the passive monetary regime which had occurred before the nomination of Paul Volcker in $1979\left(\alpha_{2}=0.89\right.$ and $\left.\gamma_{2}=0.15\right)$.

In addition, we assume that probabilities of switching are as follows:

$$
\begin{gathered}
p_{11}=0.95, \\
p_{22}=0.8-\lambda \pi_{t-1}^{2} .
\end{gathered}
$$

We compare two cases. In the first case, we consider an exogenous regime switching monetary policy, i.e. we assume that probabilities of switching are constant, $\lambda=0$. In the second case, we consider an endogenous regime switching economy with $\lambda=100$. This value of $\lambda$ implies that, when inflation is at the steady state, the probability of remaining in the dovish regime is 0.8 , while this probability is equal to 0.79 when quarter to quarter inflation is $1 \%$ and 0.64 when inflation is $4 \%$. Even if the value of $\lambda$ appears to be large, induced fluctuations of probabilities are relatively small. Most parameters are calibrated as in Davig and Leeper (2007): $\beta=0.99, \sigma=1, \kappa=0.17$. Persistence and standard deviation of shocks are calibrated to generate plausible order of magnitudes of fluctuations: $\rho=0.9, \sigma_{1}=0.65 \%$ and $\sigma_{2}=0.35 \%$.

\subsection{Stabilizing properties of endogenous regime switching}

Figures 5 and 6 display the average responses of inflation and output, to a supply and a demand shocks, taking into account the possibility of future switches. We consider the exogenous and the endogenous regime switching models. For both shocks and models, we depict the average responses of output and inflation triggered by demand and supply shocks given the initial regime. As we cannot rely on the certainty equivalence, we compute the average response to the shock over 100000 different draws of shocks and regimes history. These responses take into account the two effects mentioned in section 5: the endogenous formation effect and the selection effect.

After a positive demand shock (a natural real interest shock), endogenous regime switching tends to stabilize inflation and output compared to the exogenous regime switching case in the dovish regime. The expectations of switches toward a more aggressive monetary policy lower inflation expectations, and hence, reduce incentives for firms that can re-optimize their prices to increase prices today. This limits contemporaneous inflationary pressures, increases real ex-ante interest rate and thus reduces also output through the IS curve. In this case, the state dependent probability acts as if the current monetary policy would be more aggressive than what it is actually. 
The change in the transmission mechanism due to the endogenous transition probabilities differs when considering that the initial central banker is active. The inflation rate is also smaller than its level in the exogenous regime switching case. Expectations of a potential future switch leads to smaller expected inflation (see the distributions of 1-period ahead inflation in Figure 7) and hence less inflationary pressures because inflation in the dovish regime is smaller than in the exogenous regime switching case. Although contemporaneous ex-ante real interest rate is lower, a demand shock implies a slightly higher contemporaneous output response. The reason is linked to higher expected output linked to future lower real interest rate. The difference between output responses in the two cases however appears to be modest compared to differences mentioned above.

The response of inflation to a negative supply shock (or a positive markup shock), is always smaller than the response in the exogenous regime switching model, whatever the initial regime. Thus, endogeneity succeeds in stabilizing inflation for both shocks and in each regime. When the initial monetary regime is active, the response of output is not substantially modified by endogeneity. However, when the initial regime is passive, the impact of a supply shock on output is significantly larger when transition probabilities are endogenous. A higher probability of switching toward the active regime induces a larger response of output to supply shock, reflecting the usual trade-off generated by supply shocks.

In conclusion, when inflation deviates from the targeted value, the probability of switching toward a more aggressive monetary policy increases, which tends to stabilize inflation in both regimes, independently of the source of disturbances. Similarly to the Fisherian model, the monetary policy is all the more stabilizing that the shocks are large. Contrary to the univariate case, endogeneity can be destabilizing and amplifies negative supply shocks, notably in the passive regime.

\subsection{Consequences on distributions}

As in the Fisherian case, section 5, the assumption of endogenous regime switching breaks down the property of certainty equivalence and thus alters the distributional properties of endogenous variables. Figures 7 and 8 display the distribution of inflation and output one quarter after a 3 standard deviation shock. Figure 7, respectively figure 8 represents the distribution of endogenous variables when the initial monetary policy regime is active, respectively passive. The distributions in the endogenous regime switching case are represented in red, the distributions in the exogenous regime switching case in black. Average responses are depicted by a vertical line.

First, all the distributions are tighter in the endogenous regime switching model indicating that the form of the endogeneity introduced here improves the predictability of the economy. Second, the differences induced by endogeneity are larger in the passive regime, resulting from the assumption that only the probabilities of switching from this regime to the others are state-dependent. Third, the largest impact concerns inflation. Endogeneity substantially modifies the variance of the inflation distribution consistently with the implicit objective of such state-dependent probabilities, i.e. to stabilize inflation and especially when facing large 
shocks. Fourth, we notice in Figure 7 that the endogeneity of transition probabilities reduces the occurrence of large inflation deviation by limiting the joint probability of large shocks and passive monetary policy. Finally, as in the Fisherian model, the endogeneity of transition probabilities triggers asymmetric inflation distribution. The one period ahead distribution of inflation is left-tailed after a large supply shock, resulting from the small joint probability of new positive inflationary shocks and switching toward the passive monetary policy regime.

\section{Conclusion}

In this paper, we study non-linear rational expectations models, in which shocks or regimes may follow state-dependent probability distributions. In such models, we show how to use the perturbation approach and provide the determinacy conditions as well as Taylor expansions of the solution. Based on algebraic solving, our methodology can be applied to any non linear rational expectations model, as long as there is no backward looking component.

We apply our findings to a Fisherian model of inflation determination and a New Keynesian model in which monetary policy switches endogenously between a passive and an active monetary policy regimes. Precisely, we assume that the more active regime is more likely when inflation deviates from its targeted level. This endogeneity does not affect the determinacy conditions as we assume small fluctuations of probabilities.

We identify two effects peculiar to endogenous regime switching. First, the expectations of future switches alters the economic dynamics and their size depend on the size of the shocks. We call this effect the endogenous expectations formation effect. Second, the average occurrence of each regime depends on the size of the shocks also. We call this latter effect, the endogenous selection effect. In our illustrations, both effects contribute to stabilize inflation but can destabilize output when a supply shock hits the economy. Furthermore, the two effects tend to change the variance and the skewness of economic variables' distributions depending on shocks hitting the economy. Although our examples are mainly qualitative, they illustrate that the asymmetry observed in empirical distributions could be explained through these two effects. 


\section{APPENDIX}

Sections A to $\mathrm{F}$ are for on-line publication only.

\section{A Preliminaries}

\section{A.1 Measures $\mu_{\gamma}$}

This subsection provides some useful properties of the core measure $\mu_{\gamma}$. This measure is defined in Equation and We consider a stochastic process, $u=[s, v]$ with the following conditional probability distribution (Equation 2):

$$
\forall(s, v) \in U, \quad \mu_{\gamma}\left(s, v, \phi, u^{t-1}\right)=h^{s}\left(v, \phi, u^{t-1}\right) p_{\gamma}^{s}\left(\phi, u^{t-1}\right)
$$

In addition, we assume that:

- $p_{\gamma}^{i}$ is $C^{1}$ according to its first argument and $\gamma \mapsto p_{\gamma}^{i}$ is $C^{1}$.

- For any $\phi \in \mathcal{B}$ and $u^{t-1} \in U^{\infty}, h^{i}\left(\cdot, \phi, u^{t-1}\right)$ is a normalized integrable function on $V$.

$$
\int_{V} h^{i}\left(u, \phi, u^{t-1}\right) d u=1 .
$$

- For any $u^{t} \in U^{\infty}, \phi \mapsto h^{i}\left(v, \phi, u^{t-1}\right)$ is $C^{1}$.

The measure $\mu_{\gamma}\left(\cdot, \phi, u^{t-1}\right)$ can be seen as a continuous linear form on continuous and bounded functions space, $\mathcal{B}$, and we write abusively $\int_{U} \mu_{\gamma}\left(u, \phi, u^{t-1}\right) \Psi(u) d u=<\Psi, \mu_{\gamma}>$ even if this measure is not Lebesgue-continuous in general.

\section{Lemma 1.}

1. For any $\Psi \in \mathcal{B}$, for any $\gamma \in[0,1]$ and for any $u^{t-1} \in U^{\infty}, \phi \mapsto \int_{U} \Psi(u) \mu_{\gamma}\left(u, \phi, u^{t-1}\right) d u$ is $C^{1}$.

We abusively denote by $\int_{U} \Psi(u) D_{\phi} \mu_{\gamma}\left(u, \phi, u^{t-1}\right)(\hat{\phi}) d u$ its differential at $\phi$ applied to $\hat{\phi}$.

2. It exists $C>0$, such that for any $\Psi$ in $\mathcal{B}$ for any $\gamma \in[0,1]$, for any $u^{t-1} \in U^{\infty}$, for any $\phi$ and $\hat{\phi}$ of norm equal to 1 ,

$$
\left|\int_{U} \Psi(u) D_{\phi} \mu_{\gamma}\left(u, \phi, u^{t-1}\right)(\hat{\phi}) d u\right| \leq C\|\Psi\|_{\infty}
$$

3. For any $\Psi \in \mathcal{B}$, for any $\phi$ and for any $u^{t-1} \in U^{\infty}, \gamma \mapsto \int_{U} \Psi(u) \mu_{\gamma}\left(u, \phi, u^{t-1}\right) d u$ is $C^{1}([0,1])$.

Proof. We first check that $\mu_{\gamma}$ satisfies 1. Fix $\Psi \in \mathcal{B}, \gamma \in[0,1]$ and $u^{t-1} \in U^{\infty}$, we compute:

$$
<\Psi, \mu_{\gamma}\left(u, \phi, u^{t-1}\right)>=\sum_{i=1}^{N} p_{\gamma}^{i}\left(\phi, u^{t-1}\right) \int_{V} h^{i}\left(v, \phi, u^{t-1}\right) \Psi([i \quad v]) d v .
$$


Thus, $\phi \mapsto<\Psi, \mu_{\gamma}\left(u, \phi, u^{t-1}\right)>$ is derivable because this function is the sum of the product of derivable function and an integral which is $C^{1}$ by dominated convergence theorem.

Then, we check that 2 . is satisfied.

$$
<\Psi, D_{\phi} \mu_{\gamma}\left(u, \phi, u^{t-1}\right)(\hat{\phi})>=\sum_{i=1}^{N} D_{\phi} p_{\gamma}^{i}\left(\phi, u^{t-1}\right)(\hat{\phi}) \int_{V} D_{\phi} h^{i}\left(v, \phi, u^{t-1}\right)(\hat{\phi}) \Psi([i \quad v]) d v
$$

and

$$
\left|<\Psi, D_{\phi} \mu_{\gamma}\left(u, \phi, u^{t-1}\right)(\hat{\phi})>\right| \leq\left(\sum_{i=1}^{N} \sum_{i=1}^{N} \sup \left\|\left|D_{\phi} p_{\gamma}^{i}(., .)\right|\right\| \sup \|\| D_{\phi} h_{\gamma}(., ., .) \mid \|\right)\|\Psi\|_{\infty}
$$

Finally, we verify 3 .

$$
<\Psi, \mu_{\gamma}\left(u, \phi, u^{t-1}\right)>=\gamma \sum_{i=1}^{N} p_{\gamma}^{i}\left(\phi, u^{t-1}\right) \int_{V} h^{i}\left(v, \phi, u^{t-1}\right) \Psi\left(\left[\begin{array}{ll}
i & v
\end{array}\right]\right) d v .
$$

As we suppose that for any $i \in[1, N], \gamma \mapsto p_{\gamma}^{i}$ is $C^{1}$, then $\gamma \mapsto<\Psi, \mu_{\gamma}\left(u, \phi, u^{t-1}\right)>$ is also $C^{1}$.

These properties imply that the model as a whole is smooth enough to apply Implicit Function Theorem. Obviously, when measures are state-invariant, these three properties are immediate for any measures. We notice that point 2. can be interpreted as the fact that $D_{\phi} \mu_{\gamma}\left(u, \phi, u^{t-1}\right)(\hat{\phi})$ is a distribution of order 0 uniformly bounded. Point 3 . guarantees that the measures $\mu_{\gamma}$ are a $C^{1}$ path between $\mu_{0}$ and $\mu_{1}$ and hence we can apply perturbation method around $\gamma=0$.

\section{A.2 Lag and lead operators}

We introduce some notations, useful for the following. Fix a function $\phi_{0} \in \mathcal{B}$, we define the operators $\mathcal{F}_{i}$, for $i \in\{1, \cdots N\}$ and $\mathcal{L}$ on $\mathcal{B}$ as follows:

$$
\begin{gathered}
\mathcal{F}: H \mapsto\left(\left(s^{t}, v^{t}\right) \mapsto \int_{V} H\left(s s^{t}, v v^{t}\right) h_{i}\left(v, \phi_{0}, s^{t}, v^{t}\right) d s d v\right), \\
\mathcal{F}_{i}: H \mapsto\left(\left(s^{t}, v^{t}\right) \mapsto \int_{V} H\left(i s^{t}, v v^{t}\right) h_{i}\left(v, \phi_{0}, s^{t}, v^{t}\right) d v\right), \\
\mathcal{L}: H \mapsto\left(\left(s^{t}, v^{t}\right) \mapsto H\left(s^{t-1}, v^{t-1}\right) .\right.
\end{gathered}
$$

Equation (30) implies that $\mathcal{F}_{i}, \mathcal{F}$ and $\mathcal{L}$ have the following straightforward properties.

1. $\mathcal{F}_{i} \mathcal{L}=1, \mathcal{F} \mathcal{L}=1$.

2. $\left\|\left|\mathcal{F}_{i}\||=1,\||\mathcal{F} \||=1\right.\right.$ and $\left.\|| \mathcal{L}\|\right|=1$.

where \|\|$\cdot \| \mid$ is the triple norm associated with the infinite norm $\|\cdot\|_{\infty}$ on $\mathcal{B}$. These two results are classical results in theory of operators on sequences. The first result is obtained 
by straightforward computation. The second follows from the fact that:

$$
\forall H \in \mathcal{B}, \quad\|\mathcal{L} H\|_{\infty}=\|H\|_{\infty}, \quad\left\|\mathcal{F}_{i} H\right\|_{\infty} \leq\|H\|_{\infty},
$$

and the last inequality is an equality if $H$ is constant.

\section{B Proof of Theorem 1}

\section{B.1 Proof of Theorem 1}

In this part, we prove Theorem 1 by applying the implicit function theorem applied to the operator $\mathcal{N}$. First, we recall the Implicit Function Theorem(IFT).

Theorem 2. [Abraham et al. (1988)] Let E, F, G be 3 Banach spaces, let $U \subset E, V \subset F$ be open and $f: U \times V \rightarrow G$ be $C^{r}, r \geq 1$. For some $x_{0} \in U, y_{0} \in V$ assume $D_{y} f\left(x_{0}, y_{0}\right)$ : $F \rightarrow G$ is an isomorphism. Then there are neighborhoods $U_{0}$ of $x_{0}$ and $W_{0}$ of $f\left(x_{0}, y_{0}\right)$ and a unique $C^{r}$ map $g: U_{0} \times W_{0} \rightarrow V$ such that, for all $(x, w) \in U_{0} \times W_{0}$

$$
f(x, g(x, w))=w .
$$

Thus, we check that:

1. $\mathcal{B}$ with the norm $\|.\|_{\infty}$, and $\mathbb{R}$ with $|$.$| are Banach spaces.$

2. $\mathcal{N}$ is $C^{1}$ on $\mathcal{B} \times(-M, M)$, where $M$ is a positive scalar.

3. $\phi_{0}$ satisfies $\mathcal{N}\left(\phi_{0}, 0\right)=0$.

4. $D_{\phi} \mathcal{N}\left(\phi_{0}, 0\right)$ is invertible.

The first point is immediate, $\mathcal{B}$ with the norm $\|\cdot\|_{\infty}$ is a Banach space as a product of Banach spaces. Point 2. results from the following lemma.

Lemma 2. $(\phi, \gamma) \mapsto \mathcal{N}(\phi, \gamma)$ is $C^{1}$ for $\phi \in \mathcal{B}$ and $\gamma \in(-M, M)$.

Proof. For any $\phi \in \mathcal{B}$, the function $\gamma \mapsto \mathcal{N}(\phi, \gamma)$ is $C^{1}$ by regularity of $p_{\gamma}^{i}$ and $f$. For the differentiability in $\phi$, we check that $\Phi \mapsto \mathcal{N}(\phi, \gamma)$ is differentiable, with continuous differential:

$$
\mathcal{N}(\phi, \gamma)=\sum_{i=1}^{N} p_{\gamma}^{i}\left(\phi, u^{t-1}\right) \int_{V} f(\cdots) h_{i}(v, \phi) d v
$$

$\phi \mapsto \int_{V} f(\cdots) h_{i}(v, \phi) d v$ is differentiable by regularity of $f, h_{i}$, and Lebesgue's dominated convergence Theorem. It results from the differentiability of $\phi \mapsto p_{\gamma}^{i}\left(\phi, u^{t-1}\right)$ that $\phi \mapsto$ $\mathcal{N}(\phi, \gamma)$ is differentiable and moreover:

$$
D_{\phi} \mathcal{N}(\phi, \gamma) H=\sum_{i=1}^{N} \partial_{1} p_{\gamma}^{i}\left(\phi, u^{t-1}\right) H\left(s^{t-1}, v^{t-1}\right) \int_{V} f(\cdots) h_{i}(v, \phi) d v
$$




$$
\begin{gathered}
+\sum_{i=1}^{N} p_{\gamma}^{i}\left(\phi, u^{t-1}\right) \int_{V} \partial_{1} f(\cdots) H\left(i s^{t}, v v^{t}\right) h_{i}(v, \phi) d v \\
+\sum_{i=1}^{N} p_{\gamma}^{i}\left(\phi, u^{t-1}\right) \int_{V} \partial_{2} f(\cdots) H\left(s^{t}, v^{t}\right) h_{i}(v, \phi) d v \\
+\sum_{i=1}^{N} p_{\gamma}^{i}\left(\phi, u^{t-1}\right) \int_{V} \partial_{3} f(\cdots) H\left(s^{t-1}, v^{t-1}\right) h_{i}(v, \phi) d v \\
+\sum_{i=1}^{N} p_{\gamma}^{i}\left(\phi, u^{t-1}\right) \int_{V} f(\cdots) H\left(s^{t-1}, v^{t-1}\right) \partial_{2} h_{i}(v, \phi) H\left(s^{t-1}, v^{t-1}\right) d v
\end{gathered}
$$

where, for a function $k, k(\cdots)=k\left(\phi\left(i s^{t}, v v^{t}\right), \phi\left(s^{t}, v^{t}\right), \phi\left(s^{t-1}, v^{t-1}\right), s_{t}, \gamma v_{t}\right)$.

Thus, $(\phi, \gamma) \mapsto D_{\phi} \mathcal{N}(\phi, \gamma) H$ is continuous on $\mathcal{B} \times(-M, M)$.

Points 3. and 4. result from assumptions of Theorem 1. We end the section by showing that the regularity of $\mu_{\gamma}$ implies that the differential of the operator behaves as the differential of an operator where the probabilities are exogenous.

Lemma 3. Under assumptions of Theorem 1, the differential $D_{\phi} \mathcal{N}\left(\phi_{0}, 0\right)$ satisfies:

$$
\begin{gathered}
D_{\phi} \mathcal{N}\left(\phi_{0}, 0\right) H \\
=<\partial_{1} f\left(\phi_{0}\left(\cdot s^{t}, \cdot v^{t}\right), \phi_{0}\left(s^{t}, v^{t}\right), \phi_{0}\left(s^{t-1}, v^{t-1}\right), 0\right) H\left(\cdot S^{t}, \cdot v^{t}\right), \mu_{0}\left(\cdot, \phi_{0}, u^{t-1}\right)>+ \\
<\partial_{2} f\left(\phi_{0}\left(\cdot s^{t}, \cdot v^{t}\right), \phi_{0}\left(s^{t}, v^{t}\right), \phi_{0}\left(s^{t-1}, v^{t-1}\right), 0\right) H\left(s^{t}, v^{t}\right), \mu_{0}\left(\cdot, \phi_{0}, u^{t-1}\right)>+ \\
<\partial_{3} f\left(\phi_{0}\left(\cdot s^{t}, \cdot v^{t}\right), \phi_{0}\left(s^{t}, v^{t}\right), \phi_{0}\left(s^{t-1}, v^{t-1}\right), 0\right) H\left(s^{t-1}, v^{t-1}\right), \mu_{0}\left(\cdot, \phi_{0}, u^{t-1}\right)>.
\end{gathered}
$$

Proof. We compute:

$$
\begin{gathered}
D_{\phi} \mathcal{N}\left(\phi_{0}, 0\right) H \\
=<\partial_{1} f\left(\phi_{0}\left(\cdot s^{t}, \cdot v^{t}\right), \phi_{0}\left(s^{t}, v^{t}\right), \phi_{0}\left(s^{t-1}, v^{t-1}\right), 0\right) H\left(\cdot S^{t}, \cdot v^{t}\right), \mu_{0}\left(\cdot, \phi_{0}, u^{t-1}\right)>+ \\
<\partial_{2} f\left(\phi_{0}\left(\cdot s^{t}, \cdot v^{t}\right), \phi_{0}\left(s^{t}, v^{t}\right), \phi_{0}\left(s^{t-1}, v^{t-1}\right), 0\right) H\left(s^{t}, v^{t}\right), \mu_{0}\left(\cdot, \phi_{0}, u^{t-1}\right)>+ \\
<\partial_{3} f\left(\phi_{0}\left(\cdot s^{t}, \cdot v^{t}\right), \phi_{0}\left(s^{t}, v^{t}\right), \phi_{0}\left(s^{t-1}, v^{t-1}\right), 0\right) H\left(s^{t-1}, v^{t-1}\right), \mu_{0}\left(\cdot, \phi_{0}, u^{t-1}\right)> \\
+<f\left(\phi_{0}\left(\cdot s^{t}, v^{t}\right), \phi_{0}\left(s^{t}, v^{t}\right), \phi_{0}\left(s^{t-1}, v^{t-1}\right), 0\right), D_{\phi} \mu_{0}\left(u, \phi_{0}, u^{t-1}\right) H\left(s^{t-1}, v^{t-1}\right)>.
\end{gathered}
$$

Since $\phi_{0}$ is such that $\mathcal{N}\left(\phi_{0}, 0\right)=0$, the last term is zero.

\section{B.2 State-dependent probability distribution in the absence of regime switching: some details}

This part is devoted to the study of model 8. We show that $\mathcal{N}$ satisfies points 1 . and 2. of Theorem 1. Here, there is no discrete part, thus we omit the dependence in $s_{t}$ and the indexation in $i$. The function $\phi_{0}$ is the constant $\phi_{0}\left(v^{t}\right)=\bar{z}$. By construction, $\phi_{0}$ satisfies:

$$
\mathcal{N}\left(\phi_{0}, 0\right)=f(\bar{z}, \bar{z}, \bar{z}, 0)=0 .
$$


Due to Lemma $2, \mathcal{N}$ is differentiable and according to Lemma 3, we compute:

$$
D_{\phi} \mathcal{N}\left(\phi_{0}, 0\right) h=\partial_{1} f(\bar{z}, \bar{z}, \bar{z}, 0) \mathcal{F} h+\partial_{2} f(\bar{z}, \bar{z}, \bar{z}, 0) h+\partial_{3} f(\bar{z}, \bar{z}, \bar{z}, 0) \mathcal{L} h .
$$

Thus, the linearized model coincides with standard linear rational expectations models with exogenous shocks. If the so-called Blanchard and Kahn conditions are satisfied for the linearized model (see section 4.1), then $D_{\phi} \mathcal{N}\left(\phi_{0}, 0\right)$ is invertible.

The invertibility of the differential given in Equation (31) is equivalent to the determinacy conditions of the following standard linear model:

$$
A E_{t+1} Z_{t}+B Z_{t}=\psi_{t},
$$

where $Z_{t}=\left[z_{t} z_{t-1}\right], \psi_{t}$ is a bounded stochastic process and matrices $A$ and $B$ depend on partial derivatives of $f$. We then consider the pencil $(A, B)$ and introduce its real generalized Schur decomposition, following Klein (2000): there exist unitary matrices $Q$ and $Z$, quasi triangular matrices $T$ and $S$ such that:

$$
A=Q T Z \quad \text { and } \quad B=Q S Z
$$

Furthermore, we rank the generalized eigenvalues such that $\left|T_{i i}\right|>\left|S_{i i}\right|$ for $i \in[1, n]$ and $\left|S_{i i}\right|>\left|T_{i i}\right|$ for $i \in[n+1,2 n]$ which is possible if and only if the number of explosive generalized eigenvalues is $n$ (Blanchard and Kahn, 1980; Klein, 2000). In this case, $D_{\phi} \mathcal{N}\left(\phi_{0}, 0\right)$ is invertible and

$$
D_{\phi} \mathcal{N}\left(\phi_{0}, 0\right)^{-1}=\left(1+Z_{22}^{-1} Z_{21} L\right)^{-1} Z_{22}^{-1}\left(1-S_{22}^{-1} T_{22} P\right)^{-1} S_{22}^{-1} Q_{12}^{\prime}
$$

where, for a matrix $M \in \mathcal{M}_{2 n}(\mathbb{R})$, we write $M$ by blocks of $\mathcal{M}_{n}(\mathbb{R})$ :

$$
M=\left[\begin{array}{ll}
M_{11} & M_{12} \\
M_{21} & M_{22}
\end{array}\right]
$$

\section{Linearizing non linear endogenous regime switching model}

In this section, we prove that linearizing the non-linear endogenous regime switching model (see Section 4.2) results in Equation (13).

We compute:

$$
\mathcal{N}(\phi, 0,0)\left(s^{t}, v^{t}\right)=\sum_{j=1}^{N} \bar{p}_{s_{t} j} \int_{V} f_{s_{t}}\left(\phi\left(j s^{t}, v v^{t}\right), \phi\left(s^{t}, v^{t}\right), 0\right) h_{s_{t}}\left(v, \phi, u^{t}\right) d v
$$


We use Lemma 3 to evaluate its differential, $D_{\phi} \mathcal{N}\left(\phi_{0}, 0\right)$ :

$$
D_{\phi} \mathcal{N}\left(\phi_{0}, 0,0\right) h\left(s^{t}\right)=\sum_{j=1}^{N} \bar{p}_{s_{t} j} \partial_{1} f_{s_{t}}\left(\bar{z}_{j}, \bar{z}_{s_{t}}, 0\right) \mathcal{F}_{j} h+\left(\sum_{j=1}^{N} \bar{p}_{s_{t} j} \partial_{2} f_{s_{t}}\left(\bar{z}_{j}, \bar{z}_{s_{t}}, 0\right)\right) h .
$$

Introducing $A_{i j}$ and $B_{i}$ defined for $(i, j) \in\{1, \cdots, N\}^{2}$ by:

$$
B_{i}=\sum_{j=1}^{N} \bar{p}_{i j} \partial_{2} f_{i}\left(\bar{z}_{j}, \bar{z}_{i}, 0\right) \quad \text { and } \quad A_{i j}=\partial_{1} f_{i}\left(\bar{z}_{j}, \bar{z}_{i}, 0\right)
$$

we get that:

$$
D_{\phi} \mathcal{N}\left(\phi_{0}, 0,0\right) h=\sum_{j=1}^{N} \bar{p}_{s_{t} j} A_{s_{t} j} \mathcal{F}_{j}+B_{s_{t}} .
$$

This proves that Equation (13) corresponds to the linearized model at $\phi_{0}$ associated with the non-linear endogenous regime switching model 10.

\section{C.1 Proof of proposition 2}

In this section, we prove Proposition 2. The proof is a refinement of Barthélemy and Marx (2012). Let us denote by $\psi$ a function in $\mathcal{B}\left(U^{\infty}\right)$, we consider the equation $D_{\phi} \mathcal{N}\left(\phi_{0}, 0,0\right) h=$ $\psi$. Then, for all $i \in\{1, \cdots N\}$,

$$
D_{\phi} \mathcal{N}\left(\phi_{0}, 0\right) h=\sum_{j=1}^{N} \bar{p}_{s_{t} j} A_{s_{t} j} \mathcal{F}_{j} h+B_{s_{t}} h=\psi .
$$

Then, introducing the operator $\mathcal{R}$, defined by:

$$
\mathcal{R}(\phi)\left(s^{t}, v^{t}\right)=-\sum_{j} p_{s_{t} j} B_{s_{t}}^{-1} A_{s_{t} j} \mathcal{F}_{j} \phi
$$

Equation (33) can be rewritten as

$$
(\mathbb{1}-\mathcal{R}) h=\tilde{\psi},
$$

where $\tilde{\psi}\left(s^{t}, v^{t}\right)=B_{s_{t}}^{-1} \psi\left(s^{t}, v^{t}\right)$. Mimicking the proof in Barthélemy and Marx (2012), we have the following result:

Lemma 4. The operator $\mathcal{R}$ is bounded and its spectrum is given by

$$
\sigma(\mathcal{R})=[-\nu, \nu]
$$

where

$$
\nu=\lim _{p \rightarrow+\infty} u_{p}
$$

The proof of this Lemma is completely similar to the proof of Lemma 4 in Barthélemy and Marx (2012) and we refer to this paper for the details. 
As a consequence, we get that $(\mathbb{1}-\mathcal{R})$ is invertible if and only if $\nu<1$. Finally, the operator $D_{\phi} \mathcal{N}\left(\phi_{0}, 0,0\right)$ is invertible if and only if the linearized model 33 is invertible. In this case, the inverse of the operator $D_{\phi} \mathcal{N}\left(\phi_{0}, 0,0\right)$ is then given by

$$
D_{\phi} \mathcal{N}\left(\phi_{0}, 0,0\right)^{-1}(\psi)=\sum_{k=0}^{\infty} \mathcal{R}^{k} \tilde{\psi}
$$

This ends the proof of Proposition 2.

\section{C.2 Proof of Proposition 3}

We first compute the fist-order expansion of the unique solution of model 10 in general and prove Equation (14).

We compute $D_{\gamma} \mathcal{N}\left(\phi_{0}, 0,0\right)$ and $D_{\sigma} \mathcal{N}\left(\phi_{0}, 0,0\right)$.

$$
\begin{aligned}
D_{\gamma} \mathcal{N}\left(\phi_{0}, 0,0\right)\left(s^{t}, v^{t}\right) & =\sum_{j=1}^{N} \partial_{2} p_{s_{t} j}\left(0, z_{s_{t}}\right) f_{s_{t}}\left(\bar{z}_{j}, \bar{z}_{s_{t}}, 0\right), \\
D_{\sigma} \mathcal{N}\left(\phi_{0}, 0,0\right)\left(s^{t}, v^{t}\right) & =\sum_{j=1}^{N} p_{s_{t} j}\left(0, z_{s_{t}}\right) \partial_{3} f_{s_{t}}\left(\bar{z}_{j}, \bar{z}_{s_{t}}, 0\right) v_{t} .
\end{aligned}
$$

Thus, by the method of undetermined coefficients, we see that

$$
D_{\phi} \mathcal{N}\left(\phi_{0}, 0,0\right)^{-1} D_{\gamma} \mathcal{N}\left(\phi_{0}, 0,0\right)\left(s^{t}, v^{t}\right)=r_{s_{t}}, \quad D_{\phi} \mathcal{N}\left(\phi_{0}, 0,0\right)^{-1} D_{\sigma} \mathcal{N}\left(\phi_{0}, 0,0\right)\left(s^{t}, v^{t}\right)=R_{s_{t}} v_{t},
$$

where

$$
\left[\begin{array}{c}
R_{1} \\
\vdots \\
R_{N}
\end{array}\right]=\left(\mathbb{1}+\Lambda^{\prime} \otimes\left(B^{-1}(P \otimes \mathbb{1}) A\right)\right)\left[\begin{array}{c}
\sum_{j} p_{1 j} \partial_{3} f_{1}\left(\bar{z}_{j}, \bar{z}_{1}, 0\right) \\
\vdots \\
\sum_{j} p_{N j} \partial_{3} f_{N}\left(\bar{z}_{j}, \bar{z}_{N}, 0\right)
\end{array}\right]
$$

and

$$
\left[\begin{array}{c}
r_{1} \\
\vdots \\
r_{N}
\end{array}\right]=-\left(\mathbb{1}+\Lambda^{\prime} \otimes\left(B^{-1}(P \otimes \mathbb{1}) A\right)\right) B^{-1}\left[\begin{array}{c}
\sum_{j} \partial_{1} p_{1 j}\left(0, \bar{z}_{1}\right) f_{1}\left(\bar{z}_{j}, \bar{z}_{1}, 0\right) \\
\vdots \\
\sum_{j} \partial_{1} p_{N j}\left(0, \bar{z}_{N}\right) \cdot f_{N}\left(\bar{z}_{j}, \bar{z}_{N}, 0\right)
\end{array}\right]
$$

where $P$ is the transition probabilities matrix when probabilities are constant, i.e. for $\gamma=0$.

We now turn to the case in which the steady state does not depend on the regime. We aim at proving Equation (15) in proposition 3.

We first notice that, if for any $i, \bar{z}_{i}=\bar{z}$, then, for any $i$,

$$
\sum_{j} \partial_{2} p_{i j}(0, \bar{z}) f_{i}(\bar{z}, \bar{z}, 0)=\partial_{2}\left(\sum_{j} p_{i j}\right)(0, \bar{z}) f_{i}(\bar{z}, \bar{z}, 0)=0
$$


since the sum of the probabilities is constant equal to 1 . Therefore, the first-order expansion in $\gamma$ is 0 . More generally, we notice that, for $k>0$ :

$$
D_{\gamma}^{k} \mathcal{N}\left(\phi_{0}, 0,0\right)\left(s^{t}, v^{t}\right)=\sum_{j=1}^{N} \partial_{2}^{k} p_{s_{t} j}(0, \bar{z}) f_{s_{t}}(\bar{z}, \bar{z}, 0)=0
$$

Thus the coefficients of $\gamma^{k}$ in the expansion of the solution are zero, for any $k>0$. We differentiate equation (7) to get that:

$$
\begin{aligned}
& D_{\phi} \mathcal{N}(\phi(\sigma, \gamma), \sigma, \gamma) \partial_{\sigma} \phi(\sigma, \gamma)+\partial_{\sigma} \mathcal{N}(\phi(\sigma, \gamma), \sigma, \gamma)=0 \\
& D_{\phi} \mathcal{N}(\phi(\sigma, \gamma), \sigma, \gamma) \partial_{\gamma} \phi(\sigma, \gamma)+\partial_{\gamma} \mathcal{N}(\phi(\sigma, \gamma), \sigma, \gamma)=0
\end{aligned}
$$

Thus,

$$
\partial_{\sigma} \phi(0,0)\left(s^{t}, v^{t}\right)=R_{s_{t}} v_{t}, \quad \partial_{\gamma} \phi(0,0)=0
$$

Differentiating once again, we get that:

$$
\begin{gathered}
0=D_{\phi, \sigma}^{2} \mathcal{N}(\phi(\sigma, \gamma), \sigma, \gamma) \partial_{\gamma} \phi(\sigma, \gamma)+D_{\phi}^{2} \mathcal{N}(\phi(\sigma, \gamma), \sigma, \gamma) \partial_{\gamma} \phi(\sigma, \gamma) \partial_{\sigma} \phi(\sigma, \gamma) \\
+D_{\phi} \mathcal{N}(\phi(\sigma, \gamma), \sigma, \gamma) \partial_{\sigma, \gamma}^{2} \phi(\sigma, \gamma)+D_{\sigma, \gamma}^{2} \mathcal{N}(\phi(\sigma, \gamma), \sigma, \gamma)+D_{\phi, \gamma}^{2} \mathcal{N}(\phi(\sigma, \gamma), \sigma, \gamma) \partial_{\sigma} \phi(\sigma, \gamma)
\end{gathered}
$$

Thus,

$$
D_{\phi} \mathcal{N}\left(\phi_{0}, 0,0\right) \partial_{\sigma, \gamma}^{2} \phi(0,0)=-D_{\phi, \gamma}^{2} \mathcal{N}\left(\phi_{0}, 0,0\right) \partial_{\sigma} \phi(0,0)
$$

This leads to

$$
\partial_{\sigma, \gamma}^{2} \phi(0,0)\left(s^{t}, v^{t}\right)=h_{s_{t}} v_{t}
$$

where

$$
\left[\begin{array}{c}
h_{1} \\
\vdots \\
h_{N}
\end{array}\right]=-\left(\mathbb{1}+\Lambda^{\prime} \otimes\left(B^{-1}(P \otimes \mathbb{1}) A\right)\right)\left[\begin{array}{c}
\sum_{j=1}^{N} \partial_{2} p_{1 j}(0, \bar{z}) R_{j} \\
\vdots \\
\sum_{j=1}^{N} \partial_{2} p_{N j}(0, \bar{z}) R_{j}
\end{array}\right]
$$

Moreover, differentiating (7) twice with respect to $\sigma$, we get that:

$$
\begin{gathered}
0=2 D_{\phi, \sigma}^{2} \mathcal{N}(\phi(\sigma, \gamma), \sigma, \gamma) \partial_{\sigma} \phi(\sigma, \gamma)+D_{\phi}^{2} \mathcal{N}(\phi(\sigma, \gamma), \sigma, \gamma) \partial_{\sigma} \phi(\sigma, \gamma) \partial_{\sigma} \phi(\sigma, \gamma) \\
+D_{\phi} \mathcal{N}(\phi(\sigma, \gamma), \sigma, \gamma) \partial_{\sigma}^{2} \phi(\sigma, \gamma)+D_{\sigma}^{2} \mathcal{N}(\phi(\sigma, \gamma), \sigma, \gamma)
\end{gathered}
$$

and :

$$
\begin{gathered}
D_{\phi} \mathcal{N}\left(\phi_{0}, 0,0\right) \partial_{\sigma}^{2} \phi(0,0)=-2 D_{\phi, \sigma}^{2} \mathcal{N}\left(\phi_{0}, 0,0\right) \partial_{\sigma} \phi(0,0)-D_{\phi}^{2} \mathcal{N}\left(\phi_{0}, 0,0\right) \partial_{\sigma} \phi(0,0) \partial_{\sigma} \phi(0,0) \\
-D_{\sigma}^{2} \mathcal{N}\left(\phi_{0}, 0,0\right)
\end{gathered}
$$

We finally get

$$
\partial_{\sigma}^{2} \phi(0,0)\left(s^{t}, v^{t}\right)=q\left(s_{t}, v_{t}\right)
$$


where $q$ satisfies

$$
D_{\phi} \mathcal{N}\left(\phi_{0}, 0,0\right) q=-2 \sum_{j=1}^{N} p_{s_{t} j} \partial_{31} f(\bar{z}, \bar{z}, 0)\left(v_{t}, R_{j} v_{t}\right)-\partial_{33} f(\bar{z}, \bar{z}, 0)\left(v_{t}, v_{t}\right)
$$

$-\sum_{j=1}^{N} p_{s_{t} j}\left[\partial_{11} f(\bar{z}, \bar{z}, 0)\left(R_{j} \Lambda v_{t}, R_{j} \Lambda v_{t}\right)+2 \partial_{12} f(\bar{z}, \bar{z}, 0)\left(R_{j} \Lambda v_{t}, R_{s_{t}} v_{t}\right)+\partial_{22} f(\bar{z}, \bar{z}, 0)\left(R_{s_{t}} v_{t}, R_{s_{t}} v_{t}\right)\right]$

\section{Proof of Proposition 4}

In this section, we prove Proposition 4. The existence of the solution relies on the results of Proposition 3. Since the model is linear, the expansion is valid for any $\sigma$. The analyticity of probabilities and of the model itself spread to the solution. Therefore, the solution can be written as:

$$
z_{t}\left(s_{t}, v_{t}\right)=\sum_{k=0}^{\infty} \gamma^{k} w_{t}^{k}\left(s_{t}, v_{t}\right)
$$

We define

$$
W_{t}^{k}=\left[\begin{array}{c}
w_{t}^{k}\left(1, v_{t}\right) \\
w_{t}^{k}\left(2, v_{t}\right)
\end{array}\right], \quad Z_{t}=\left[\begin{array}{c}
z_{t}\left(1, v_{t}\right) \\
z_{t}\left(2, v_{t}\right)
\end{array}\right]
$$

Equation (16) is rewritten as

$P\left(z_{t}, \gamma\right) \int Z_{t+1}\left(v_{t+1}\right) d \nu_{t+1}+\left[\begin{array}{cc}A_{1} & 0 \\ 0 & A_{2}\end{array}\right]^{-1}\left[\begin{array}{cc}B_{1} & 0 \\ 0 & B_{2}\end{array}\right] Z_{t}\left(v_{t}\right)+\left[\begin{array}{cc}A_{1} & 0 \\ 0 & A_{2}\end{array}\right]^{-1}\left[\begin{array}{l}C_{1} \\ C_{2}\end{array}\right] v_{t}=0$

where

$$
P\left(Z_{t}, \gamma\right)=\left[\begin{array}{cc}
p_{11}\left(z_{t}\left(1, v_{t}\right), \gamma\right) & 1-p_{11}\left(z_{t}\left(1, v_{t}\right), \gamma\right) \\
1-p_{22}\left(z_{t}\left(2, v_{t}\right), \gamma\right) & p_{22}\left(z_{t}\left(2, v_{t}\right), \gamma\right)
\end{array}\right]
$$

By fixing $\gamma$ equal to 0 , we find that $P_{0, s_{t}}=-C_{s_{t}} v_{t}$ and hence, $w_{t}^{0}$ solves a standard linear exogenous regime switching model: $w_{t}^{0}=R_{s_{t}} v_{t}$, where $R_{s_{t}}$ is defined above.

Then, we get $P_{1, s_{t}}$ by using the fact that $Z_{t}$ and $W_{t}^{0}$ satisfies similar equations:

$$
\left[P\left(Z_{t}, \gamma\right)-P\left(W_{t}^{0}, 0\right)\right] \int Z_{t+1}+P\left(W_{t}^{0}, 0\right) \int\left(Z_{t+1}-W_{t+1}^{0}\right)+\left[\begin{array}{cc}
A_{1} & 0 \\
0 & A_{2}
\end{array}\right]^{-1}\left[\begin{array}{cc}
B_{1} & 0 \\
0 & B_{2}
\end{array}\right]\left(Z_{t}-W_{t}^{0}\right)=0
$$

Then, by taking the first-order in $\gamma$ we find that $W_{t}^{1}$ satisfies:

$$
\partial_{\gamma} P\left(W_{t}^{0}, 0\right) \int W_{t+1}^{0}+P\left(W_{t}^{0}, 0\right) \int W_{t+1}^{1}+\left[\begin{array}{cc}
A_{1} & 0 \\
0 & A_{2}
\end{array}\right]^{-1}\left[\begin{array}{cc}
B_{1} & 0 \\
0 & B_{2}
\end{array}\right] W_{t}^{1}=0 .
$$

Reiterating this computation, we can compute recursively $W_{t}^{k}$ given lower order $W_{t}^{j}$. $P_{k, s_{t}}$ 
is a polynomial given by the term in $\gamma^{k}$ in:

$$
-\left[P\left(\sum_{j=1}^{k} \gamma^{j} W_{t}^{j}, \gamma\right)-P\left(\sum_{j=1}^{k-1} \gamma^{j} W_{t}^{j}, \gamma\right)\right] \int \sum_{j=1}^{k-1} \gamma^{j} W_{t+1}^{j}
$$

This leads to the following algorithm, proposing an iterative procedure to accomplish these two steps in the case of polynomial probabilities.

Let us denote by $U_{j}$, for $j \in\{1, \cdots, p(k)\}$, the canonical basis of p-order polynomial on $\mathbb{R}^{n}$ where $p(k)$ refers to the maximum degree of $P_{k, s_{t}}$ for any regime, $s_{t}$. We define by $a_{s_{t}}^{k}=\left[a_{1, s_{t}}^{k} \cdot a_{p(k), s_{t}}^{k}\right]\left(b_{s_{t}}^{k}\right.$ resp.) the coordinates of $P_{k, s_{t}}\left(w^{k}\right.$ in regime $s_{t}$ resp.) in this basis.

Algorithm 1. Set $k=0$,

1. Compute the coefficients $a_{s_{t}}^{k}$ for any regime, $s_{t}$.

2. Compute the matrix, $M^{k}=\left[M_{1}^{k} \cdot M_{p(k)}^{k}\right]^{\prime}$, representing the expectation operator in polynomial space:

$$
\int U_{j}\left(v_{t+1}\right) d \nu_{t+1}=M_{j}^{k}\left[\begin{array}{c}
U_{1} \\
\vdots \\
U_{p(k)}
\end{array}\right]
$$

3. Compute the coordinates of $w^{k}, b^{k}$ :

$$
\operatorname{vec}\left(\begin{array}{c}
b_{1}^{k} \\
\vdots \\
b_{p(k)}^{k}
\end{array}\right)=\left[\left(M^{k}\right)^{\prime} \otimes\left(\operatorname{diag}\left(\left[B_{1}^{-1} A_{1} \ldots B_{N}^{-1} A_{N}\right]\right)[\bar{P} \otimes I]\right)+I\right]^{-1} \cdot\left[I \otimes \operatorname{diag}\left(\left[B_{1}^{-1} \ldots B_{N}^{-1}\right]\right)\right] \operatorname{vec}\left(\begin{array}{c}
\alpha_{1}^{k} \\
\vdots \\
\alpha_{N}^{k}
\end{array}\right)
$$

4. Increment $k$ and go back to Step 1 until $w^{k}$ is sufficiently small.

This algorithm allows for computing the Taylor expansion in $\sigma$ of the solution $z_{t}$ at any order. The algorithm is based on results from Proposition 4 and the linearity of the linearized model. The first two steps require to compute moments of the innovation, $\nu$, and to compute the coordinates in a basis of cardinal $n k^{p}$, which requires $O\left(n k^{p}\right)$ operations. The third step consists in a purely algebraic inversion of matrix and is not very costly.

\section{E Proof of Proposition 5}

\section{E.1 Existence and uniqueness of a s.r.e.e.}

This results from Proposition 2.

$$
S_{1}=\left[\begin{array}{ll}
\frac{p_{11}}{\left|\alpha_{1}\right|} & \frac{p_{12}}{\left|\alpha_{2}\right|} \\
\frac{p_{21}}{\left|\alpha_{1}\right|} & \frac{p_{22}}{\left|\alpha_{2}\right|}
\end{array}\right]
$$

Here, $S_{p}=S_{1}^{p}$ and we only need to check that all eigenvalues of $S_{1}$ are smaller than 1 . This condition exactly coincides with Farmer et al. (2009a) determinacy condition. 
Furthermore, when $\left|\alpha_{1}\right|>p_{11}^{-}$and $\left|\alpha_{2}\right|>p_{22}^{-}$, Davig and Leeper (2007) proves that the eigenvalues of $S_{1}$ are smaller than one if and only if:

$$
\left|\alpha_{1}\right| \cdot\left|\alpha_{2}\right|+\bar{p}_{22}^{-}\left(1-\left|\alpha_{1}\right|\right)+p_{11}^{-}\left(1-\left|\alpha_{2}\right|\right)>1
$$

\section{E.2 The solution when probabilities are exogenous}

We directly apply Proposition 4 and with the notations of section D, $\Lambda=\rho$ and

$$
P=\left[\begin{array}{cc}
\frac{p_{\overline{1} 1}}{\alpha_{1}} & \frac{1-p_{\overline{1} 1}}{\alpha_{2}} \\
\frac{1-p_{\overline{2} 2}}{\alpha_{1}} & \frac{p_{\overline{2} 2}}{\alpha_{2}}
\end{array}\right] .
$$

We know that:

$$
\pi_{t}=\frac{\Lambda_{t} r_{t}}{\alpha_{t}}
$$

where

$$
\left[\begin{array}{c}
\Lambda_{1} \\
\Lambda_{2}
\end{array}\right]=(I-\rho P)^{-1}\left[\begin{array}{l}
1 \\
1
\end{array}\right]
$$

In particular, if $\rho=0, \Lambda_{1}=\Lambda_{2}=1$.

\section{E.3 Computation of $D_{\gamma} \mathcal{N}\left(\phi_{0}, 0\right)$}

The expression of $\mathcal{N}(\phi, \gamma)$ leads to:

$$
D_{\gamma} \mathcal{N}\left(\phi_{0}, 0\right)=\rho \frac{\Lambda_{s_{t}}}{\alpha_{s_{t}}}\left(\frac{\Lambda_{1}}{\alpha_{1}}-\frac{\Lambda_{2}}{\alpha_{2}}\right)\left[\frac{\Lambda_{s_{t}}}{\alpha_{s_{t}}} \lambda_{s_{t}} r_{t}\right] r_{t}^{2} .
$$

We thus define $\gamma_{i}=\frac{\Lambda_{i}^{2}}{\alpha_{i}^{2}}\left(\frac{\Lambda_{1}}{\alpha_{1}}-\frac{\Lambda_{2}}{\alpha_{2}}\right) \lambda_{i 1}$, to rewrite the differential as follows:

$$
D_{\gamma} \mathcal{N}\left(\phi_{0}, 0\right)=\rho \gamma_{s_{t}} r_{t}^{3}
$$

\section{E.4 Computation of $D_{\phi} \mathcal{N}\left(\phi_{0}, 0\right)^{-1} D_{\gamma} \mathcal{N}\left(\phi_{0}, 0\right)$}

Computing :

$$
D_{\phi} \mathcal{N}\left(\phi_{0}, 0\right)^{-1} D_{\gamma} \mathcal{N}\left(\phi_{0}, 0\right)=-\frac{r_{t} \Lambda_{s_{t}}}{\alpha_{s_{t}}}+\gamma \rho \sum_{k=0}^{\infty} \mathbb{E}_{t} \frac{\gamma_{t+k}}{\prod_{j=0}^{k} \alpha_{t+j}} r_{t+k}^{3} .
$$

As $r_{t}$ is independent from the switching process,

$$
D_{\phi} \mathcal{N}\left(\Phi_{0}, 0\right)^{-1} D_{\gamma} \mathcal{N}\left(\phi_{0}, 0\right)=-\frac{r_{t} \Lambda_{s_{t}}}{\alpha_{s_{t}}}+\gamma \rho \sum_{k=0}^{\infty} \mathbb{E}_{t} \frac{\gamma_{t+k}}{\prod_{j=0}^{k} \alpha_{t+j}} \mathbb{E}_{t} r_{t+k}^{3}
$$

We notice that:

$$
\mathbb{E}_{t} \frac{\gamma_{t+k}}{\prod_{j=0}^{k} \alpha_{t+j}}=\frac{1}{\alpha_{s_{t}}} \tilde{P}_{s_{t}}^{k}\left[\begin{array}{c}
\gamma_{1} \\
\gamma_{2}
\end{array}\right]
$$


Now we compute first and second raw moments of $r_{t}$ :

$$
\begin{gathered}
\mathbb{E}_{t} r_{t+k}^{3}=3 \operatorname{var}(v) \mathbb{E}_{t} r_{t+k-1}+\rho^{3} \mathbb{E}_{t} r_{t+k-1}^{3}, \\
\mathbb{E}_{t} r_{t+k}^{3}=3 \operatorname{var}(v) r_{t} \sum_{i=1}^{k}\left(\rho^{3}\right)^{i-1} \rho^{k+1-i}+\left(\rho^{3}\right)^{k} r_{t}^{3}, \\
\mathbb{E}_{t} r_{t+k}^{3}=3 \rho^{k} \frac{1-\left(\rho^{2}\right)^{k}}{1-\rho^{2}} \operatorname{var}(v) r_{t}+\left(\rho^{3}\right)^{k} r_{t}^{3}, \\
\mathbb{E}_{t} r_{t+k}^{3}=3 \frac{\rho^{k}}{1-\rho^{2}} \operatorname{var}(v) r_{t}+\left(\rho^{3}\right)^{k}\left[r_{t}^{3}-\frac{\operatorname{var}(v) r_{t}}{1-\rho^{2}}\right] .
\end{gathered}
$$

Thus,

$$
\begin{gathered}
\gamma \rho \sum_{k=0}^{\infty} \mathbb{E}_{t} \frac{\gamma_{t+k}}{\prod_{j=0}^{k} \alpha_{t+j}} \mathbb{E}_{t} r_{t+k}^{3}=\gamma \frac{\rho}{\alpha_{s_{t}}} \frac{\operatorname{var}(v) r_{t}}{1-\rho^{2}} \sum_{k=0}^{\infty} \rho^{k} \tilde{P}_{s_{t}}^{k}\left[\begin{array}{c}
\gamma_{1} \\
\gamma_{2}
\end{array}\right]+\left(r_{t}^{3}-\frac{\operatorname{var}(v) r_{t}}{1-\rho^{2}}\right) \sum_{k=0}^{\infty} \rho^{3 k} \tilde{P}_{s_{t}}^{k}\left[\begin{array}{c}
\gamma_{1} \\
\gamma_{2}
\end{array}\right] \\
\gamma \rho \sum_{k=0}^{\infty} E_{t} \frac{\gamma_{t+k}}{\prod_{j=0}^{k} \alpha_{t+j}} \mathbb{E}_{t} r_{t+k}^{3}=\gamma \frac{\rho}{\alpha_{s_{t}}} \frac{\operatorname{var}(v) r_{t}}{1-\rho^{2}}(I-\rho \tilde{P})^{-1}\left[\begin{array}{c}
\gamma_{1} \\
\gamma_{2}
\end{array}\right]+\left(r_{t}^{3}-\frac{\operatorname{var}(v) r_{t}}{1-\rho^{2}}\right)\left(I-\rho^{3} \tilde{P}\right)^{-1}\left[\begin{array}{c}
\gamma_{1} \\
\gamma_{2}
\end{array}\right]
\end{gathered}
$$

Finally by applying Proposition 4 we find:

$$
\pi_{t}=-\frac{r_{t} \Lambda_{s_{t}}}{\alpha_{s_{t}}}+\rho \gamma\left(c_{s_{t}} r_{t}^{3}+d_{s_{t}} \frac{\operatorname{var}(v)}{1-\rho^{2}} r_{t}\right)+o(\gamma)
$$

where

$$
\begin{gathered}
{\left[\begin{array}{l}
c_{1} \\
c_{2}
\end{array}\right]=A^{-1}\left(I-\rho^{3} \tilde{P}\right)^{-1}\left[\begin{array}{l}
\gamma_{1} \\
\gamma_{2}
\end{array}\right]} \\
{\left[\begin{array}{l}
d_{1} \\
d_{2}
\end{array}\right]=A^{-1}\left[(I-\rho \tilde{P})^{-1}-\left(I-\rho^{3} \tilde{P}\right)^{-1}\right]\left[\begin{array}{l}
\gamma_{1} \\
\gamma_{2}
\end{array}\right] .}
\end{gathered}
$$

\section{F The New-Keynesian model}

The model is :

$$
\begin{gathered}
\pi_{t}=\kappa x_{t}+\beta \mathbb{E}_{t} \pi_{t+1}+u_{t}, \\
x_{t}=\mathbb{E}_{t}\left(x_{t+1}\right)-\sigma\left(\alpha_{s_{t}} \pi_{t}+\gamma_{s_{t}} x_{t}-\mathbb{E}_{t} \pi_{t+1}\right)+v_{t},
\end{gathered}
$$

with following transition probabilities:

$$
p_{i j}\left(\pi_{t-1}\right)=\bar{p}_{i j}+\gamma \lambda_{i j}\left(\pi_{t-1}\right)^{2} .
$$

The persistence matrix of the shocks satisfies:

$$
\Lambda=\left[\begin{array}{cc}
\rho_{u} & 0 \\
0 & \rho_{v}
\end{array}\right] .
$$


Defining $Z_{t}=\left[\begin{array}{c}x_{t} \\ \pi_{t}\end{array}\right]$, the model can be rewritten as :

$$
\mathbb{E}_{t}\left(Z_{t+1}\right)+\Gamma_{s_{t}} Z_{t}+C_{s_{t}}\left[\begin{array}{c}
u_{t} \\
v_{t}
\end{array}\right]
$$

where

$$
\Gamma_{i}=\left[\begin{array}{cc}
1 & \sigma \\
0 & \beta
\end{array}\right]^{-1}\left[\begin{array}{cc}
-1-\sigma \gamma_{i} & -\sigma \alpha_{i} \\
\kappa & -1
\end{array}\right]
$$

and

$$
C_{i}=\left[\begin{array}{ll}
1 & \sigma \\
0 & \beta
\end{array}\right]^{-1}\left[\begin{array}{ll}
1 & 0 \\
0 & 1
\end{array}\right]
$$

We define $\Gamma=\operatorname{diag}\left(\Gamma_{1}, \Gamma_{2}\right)$. The solution of the linear exogenous model is given by

$$
Z_{t}^{0}=R_{s_{t}}\left[\begin{array}{c}
u_{t} \\
v_{t}
\end{array}\right]
$$

where

$$
\operatorname{Vect}\left[\begin{array}{l}
R_{1} \\
R_{2}
\end{array}\right]=\left[\Lambda^{\prime} \otimes(P \otimes \mathbb{1})+\mathbb{1} \otimes \Gamma\right]^{-1} \operatorname{Vect}\left[\begin{array}{l}
C_{1} \\
C_{2}
\end{array}\right] .
$$

According to Proposition 4, we know that the solution is given by $Z_{t}=Z_{t}^{0}+w_{t}$, where

$$
\mathbb{E}_{t}^{0} w_{t+1}+\Gamma_{s_{t}} w_{t}=-\lambda_{s_{t} 1}\left(\left[\begin{array}{ll}
0 & 1
\end{array}\right] R_{s_{t}}\left[\begin{array}{c}
u_{t} \\
v_{t}
\end{array}\right]\right)^{2}\left(R_{1}-R_{2}\right) \Lambda\left[\begin{array}{c}
u_{t} \\
v_{t}
\end{array}\right] .
$$

Furthermore,

$$
-\lambda_{s_{t} 1}\left(\left[\begin{array}{ll}
0 & 1
\end{array}\right] R_{s_{t}}\left[\begin{array}{c}
u_{t} \\
v_{t}
\end{array}\right]\right)^{2}\left(R_{1}-R_{2}\right) \Lambda\left[\begin{array}{c}
u_{t} \\
v_{t}
\end{array}\right]=\Sigma_{s_{t}} u_{t}^{3}+\Upsilon_{s_{t}} v_{t}^{3}+\Psi_{s_{t}} u_{t}^{2} v_{t}+\Xi_{s_{t}} u_{t} v_{t}^{2},
$$

with

$\Sigma_{s_{t}}=-\lambda_{s_{t} 1} R_{s_{t}}(2,1)^{2} \Gamma_{s_{t}}^{-1}\left(R_{1}-R_{2}\right) \Lambda\left[\begin{array}{l}1 \\ 0\end{array}\right], \quad \Upsilon_{s_{t}}=-\lambda_{s_{t} 1} R_{s_{t}}(2,2)^{2} \Gamma_{s_{t}}^{-1}\left(R_{1}-R_{2}\right) \Lambda\left[\begin{array}{l}0 \\ 1\end{array}\right]$,

and

$$
\begin{aligned}
& \Psi_{s_{t}}=-\lambda_{s_{t}} R_{s_{t}}(2,1)^{2} \Gamma_{s_{t}}^{-1}\left(R_{1}-R_{2}\right) \Lambda\left[\begin{array}{l}
0 \\
1
\end{array}\right]-2 \lambda_{s_{t}} R_{s_{t}}(2,1) R_{s_{t}}(2,2) \Gamma_{s_{t}}^{-1}\left(R_{1}-R_{2}\right) \Lambda\left[\begin{array}{l}
1 \\
0
\end{array}\right] \\
& \Xi_{s_{t}}=-\lambda_{s_{t} 1} R_{s_{t}}(2,2)^{2} \Gamma_{s_{t}}^{-1}\left(R_{1}-R_{2}\right) \Lambda\left[\begin{array}{l}
1 \\
0
\end{array}\right]-2 \lambda_{s_{t} 1} R_{s_{t}}(2,1) R_{s_{t}}(2,2) \Gamma_{s_{t}}^{-1}\left(R_{1}-R_{2}\right) \Lambda\left[\begin{array}{l}
0 \\
1
\end{array}\right] .
\end{aligned}
$$

We define $\Sigma=\operatorname{diag}\left(\Sigma_{1}, \Sigma_{2}\right), \Upsilon=\operatorname{diag}\left(\Upsilon_{1}, \Upsilon_{2}\right), \Psi=\operatorname{diag}\left(\Psi_{1}, \Psi_{2}\right)$, and $\Xi=\operatorname{diag}\left(\Xi_{1}, \Xi_{2}\right)$. 
By identification, we get that the solution $w_{t}$ is given by:

$$
w_{t}=f_{s_{t}} u_{t}^{3}+g_{s_{t}} u_{t}+h_{s_{t}} v_{t}^{3}+j_{s_{t}} v_{t}+k_{s_{t}} u_{t}^{2} v_{t}+l_{s_{t}} u_{t} v_{t}^{2},
$$

where

$$
\begin{aligned}
& {\left[\begin{array}{l}
f_{1} \\
f_{2}
\end{array}\right]=\left(\mathbb{1}+\rho_{u}(P \otimes \mathbb{1}) \Gamma^{-1}\right)^{-1} \Sigma,} \\
& {\left[\begin{array}{l}
h_{1} \\
h_{2}
\end{array}\right]=\left(\mathbb{1}+\rho_{v}(P \otimes \mathbb{1}) \Gamma^{-1}\right)^{-1} \Upsilon} \\
& {\left[\begin{array}{l}
g_{1} \\
g_{2}
\end{array}\right]=\frac{3 \sigma_{u}^{2}}{1-\rho_{u}^{2}}\left[\left(\mathbb{1}+\rho_{u}(P \otimes \mathbb{1}) \Gamma^{-1}\right)^{-1}-\left(\mathbb{1}+\rho_{u}^{3}(P \otimes \mathbb{1}) \Gamma^{-1}\right)^{-1}\right] \Upsilon} \\
& +\frac{\sigma_{v}^{2}}{1-\rho_{v}^{2}}\left[\left(\mathbb{1}+\rho_{u}(P \otimes \mathbb{1}) \Gamma^{-1}\right)^{-1}-\left(\mathbb{1}+\rho_{v}^{2} \rho_{u}(P \otimes \mathbb{1}) \Gamma^{-1}\right)^{-1}\right] \Xi \\
& {\left[\begin{array}{l}
j_{1} \\
j_{2}
\end{array}\right]=\frac{3 \sigma_{v}^{2}}{1-\rho_{v}^{2}}\left[\left(\mathbb{1}+\rho_{v}(P \otimes \mathbb{1}) \Gamma^{-1}\right)^{-1}-\left(\mathbb{1}+\rho_{v}^{3}(P \otimes \mathbb{1}) \Gamma^{-1}\right)^{-1}\right] \Sigma,} \\
& +\frac{\sigma_{u}^{2}}{1-\rho_{u}^{2}}\left[\left(\mathbb{1}+\rho_{v}(P \otimes \mathbb{1}) \Gamma^{-1}\right)^{-1}-\left(\mathbb{1}+\rho_{u}^{2} \rho_{v}(P \otimes \mathbb{1}) \Gamma^{-1}\right)^{-1}\right] \Psi \\
& {\left[\begin{array}{l}
k_{1} \\
k_{2}
\end{array}\right]=\left(\mathbb{1}+\rho_{u}^{2} \rho_{v}(P \otimes \mathbb{1}) \Gamma^{-1}\right)^{-1} \Psi,} \\
& {\left[\begin{array}{l}
l_{1} \\
l_{2}
\end{array}\right]=\left(\mathbb{1}+\rho_{v}^{2} \rho_{u}(P \otimes \mathbb{1}) \Gamma^{-1}\right)^{-1} \Xi}
\end{aligned}
$$

\section{G Numerical application}

\begin{tabular}{|c|c|}
\hline Parameter & Value \\
\hline$p_{11}^{-}$ & 0.8 \\
$p_{22}^{-}$ & 0.95 \\
$\rho$ & 0.9 \\
$\sqrt{\operatorname{var}(v)}$ & $0.25 \%$ \\
$\alpha_{1}$ & 0.9 \\
$\alpha_{2}$ & 3 \\
$\lambda_{11}$ & -40 \\
$\lambda_{22}$ & 0 \\
$\gamma$ & 1 \\
\hline
\end{tabular}

Table 1: Parameters 


\section{H Figures}

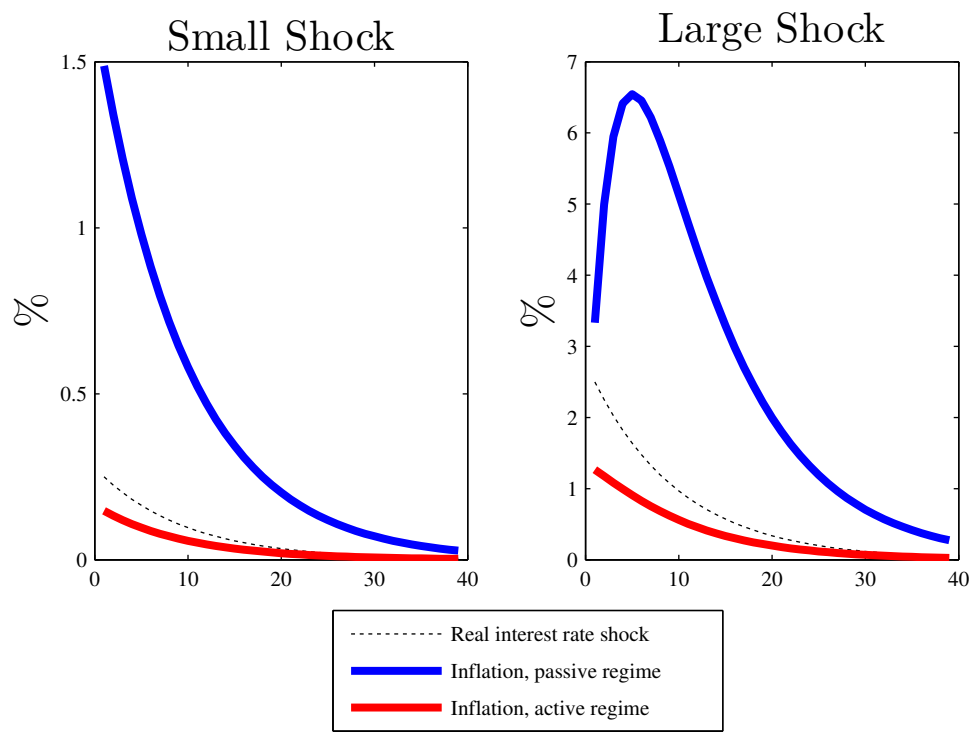

Figure 1: Responses to a real interest rate shock when regimes do not change

Note: The left (resp. right) figure depicts the responses to a 1- (resp. a 10-) standard deviation real interest shock. In dashed black line, we report the trajectory of the shock. This is not a perfect foresight equilibrium, rational agents expect future shocks. In thick blue (resp. red) line, we plot the response of inflation in the passive (resp. active) monetary policy regime. 


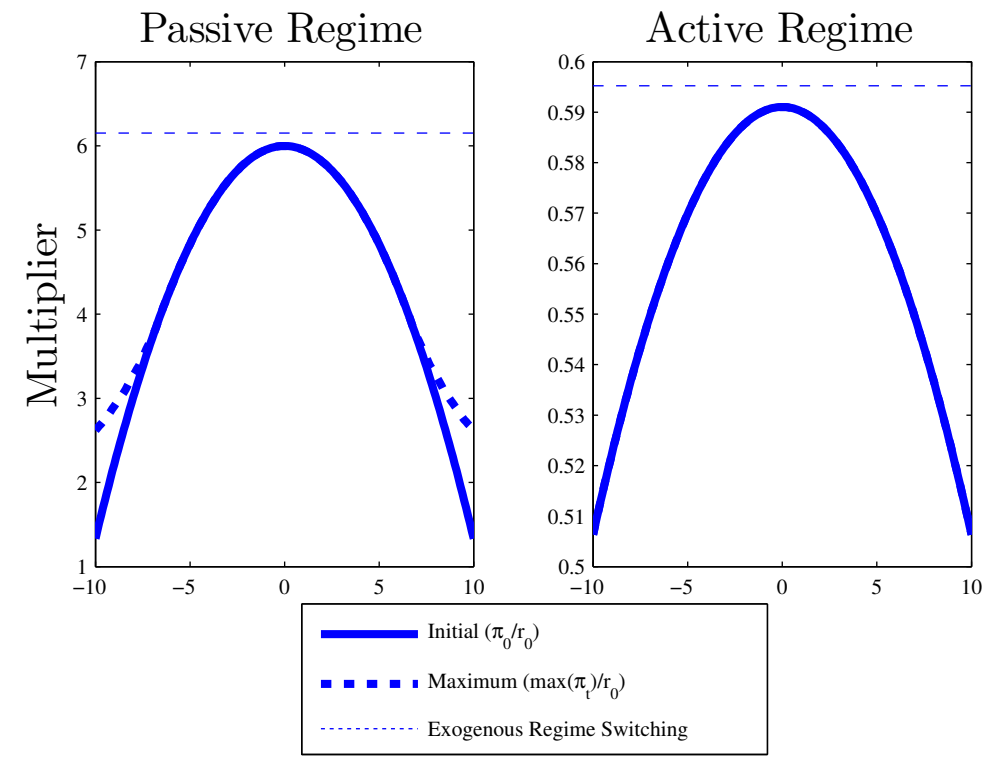

Figure 2: Multiplier with respect to the size of the shock

Note: The left (resp. right) panel depicts multipliers - the ratio of the inflation response over the size of the shock - for different size of the shock (in abscises, expressed in standard deviation) for the active regime (resp. the passive regime). The thin dashed lines report the multipliers for the exogenous regime switching $(\gamma=0)$. The thick blue curves represent the initial multiplier while the thick dashed curves represent the maximum multiplier both for the endogenous regime switching. 


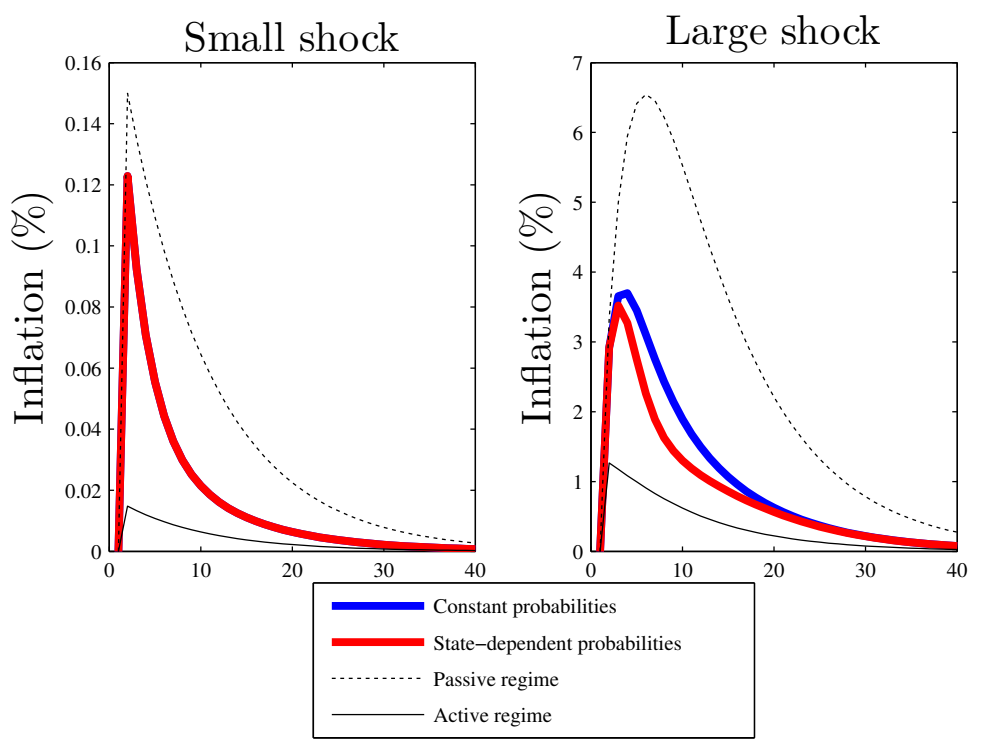

Figure 3: Responses to a real interest rate shock when regimes change

Note: The left (resp. right) figure depicts the responses to a 1- (resp. a 10-) standard deviation real interest shock. Thin black and thin dashed black lines display the responses when the monetary policy regime remains active and passive. In blue, we plot the trajectory which would prevail if the equilibrium consisted of a Markov chain with constant probabilities between the two responses. This trajectory has no economic sense but allows isolating the selection effect due to changing probabilities but not due to expectations only. In red, the expected response when probabilities are state dependent but no shocks affect the economy. We assume that the initial monetary policy regime is passive. 

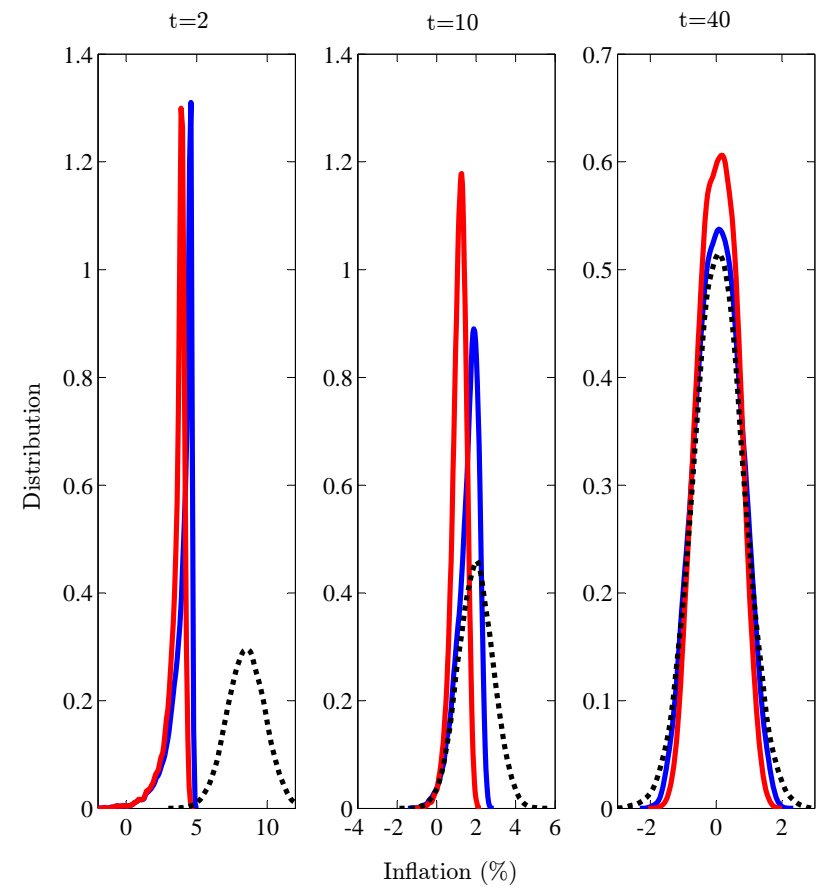

Figure 4: Distributions of inflation after a real interest rate shock

Note: We simulate 10000 trajectories after an initial 10-standard deviation shock and starting from the passive monetary policy regime. At each period a real interest rate shock hit the economy following a Gaussian distribution. In dashed black, the distribution of inflation when regimes switch exogenously. The blue curve displays the distribution if agents take into account the endogeneity of regime switching but regimes siwtch exogenously. In red, we depict the distribution in the full endogenous regime switching model. 

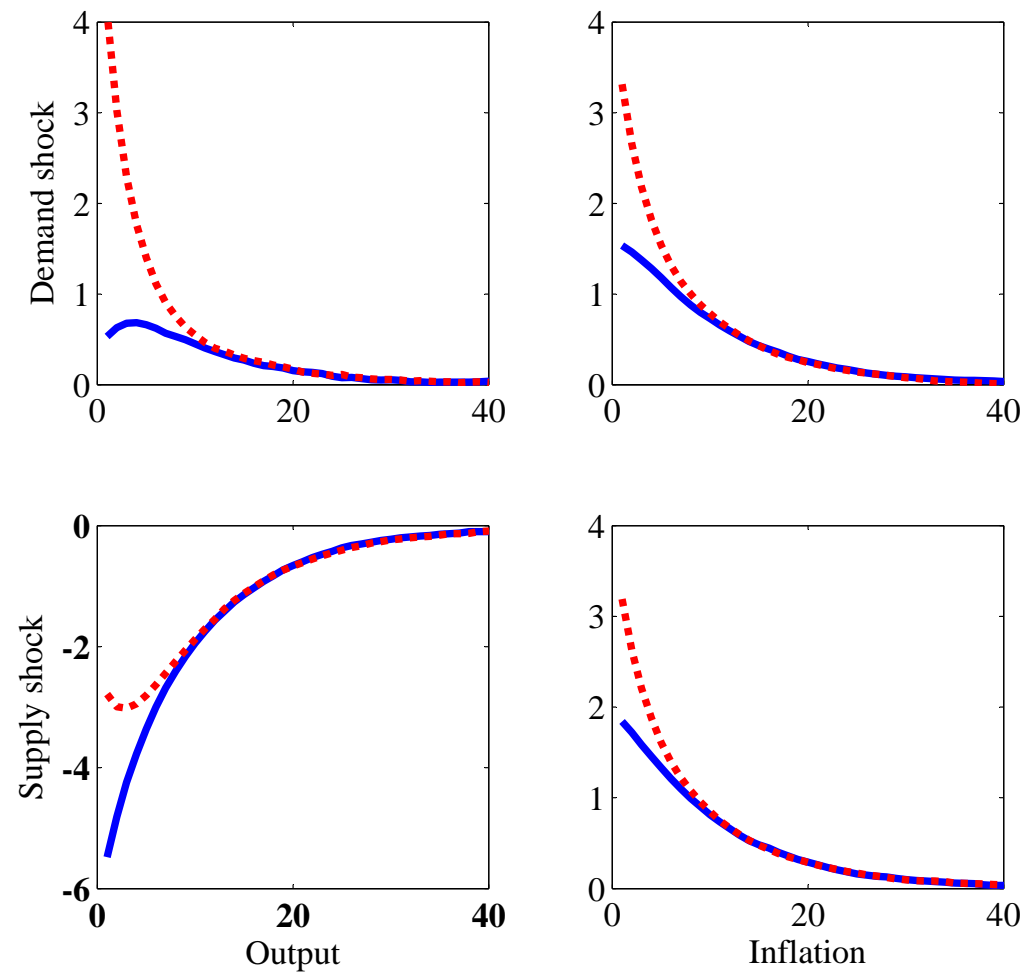

Figure 5: Impulse Response Function in the exogenous regime switching new-Keynesian model

Note: expected response of an unexpected 3 standard deviation shock to output and inflation. These graphs have been computed setting $\lambda=0$. Blue lines display the average responses (taking into account possibility of switches and shocks) when the initial monetary regime is active $\left(s_{0}=1\right)$. Red lines show the average responses when the initial monetary regime is passive $\left(s_{0}=2\right)$. The IRFs are computed by averaging 100000 random trajectories. 

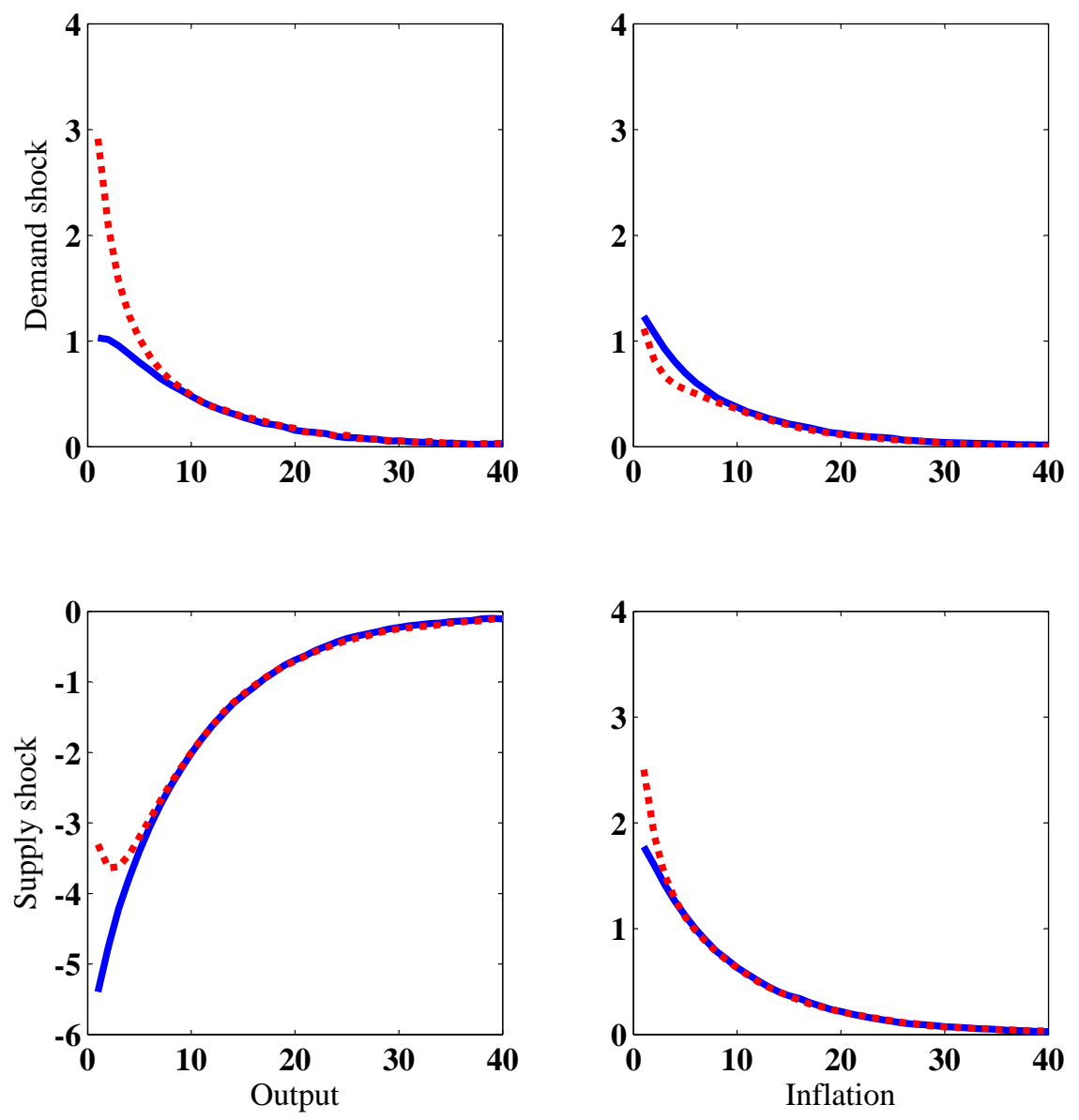

Figure 6: Impulse Response Function in the endogenous regime switching new-Keynesian model

Note: expected response of an unexpected 3 standard deviation shock to output and inflation. These graphs have been computed setting $\lambda=100$. Blue lines display the average responses (taking into account possibility of switches and shocks) when the initial monetary regime is active $\left(s_{0}=1\right)$. Red lines show the average responses when the initial monetary regime is passive $\left(s_{0}=2\right)$. The IRFs are computed by averaging 100000 random trajectories. 

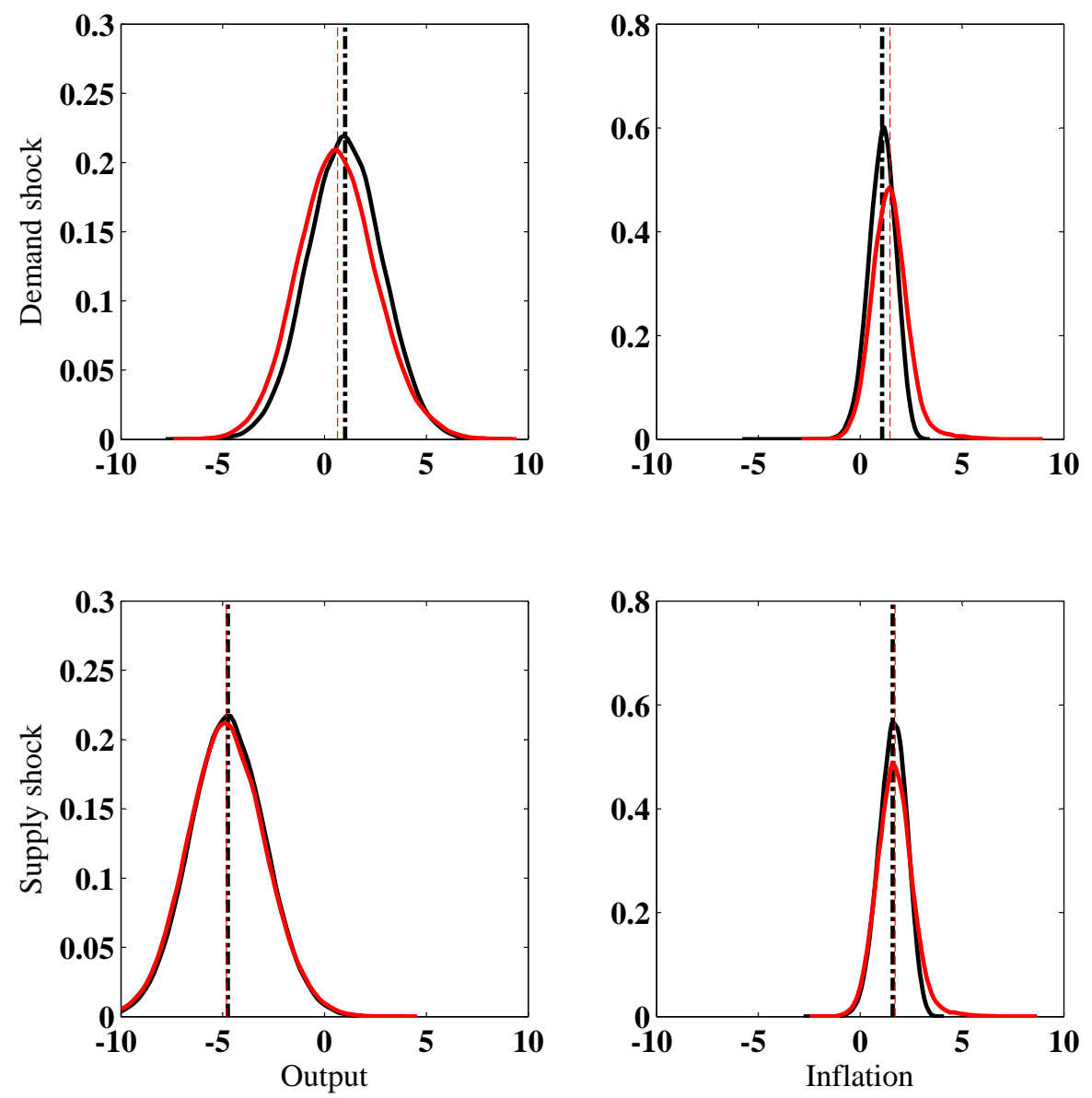

Figure 7: Distributions one period ahead when initial monetary regime is active

Note: Red (resp. black) line plots the distribution of output and inflation one period after a 3-standard deviation shock for endogenous (resp. exogenous) regime switching model. Initial monetary policy regime is active. Distributions result from 100000 draws of future shocks and regimes and are approximated using a standard kernel estimate. The vertical red and black lines report the mean of the distributions. 

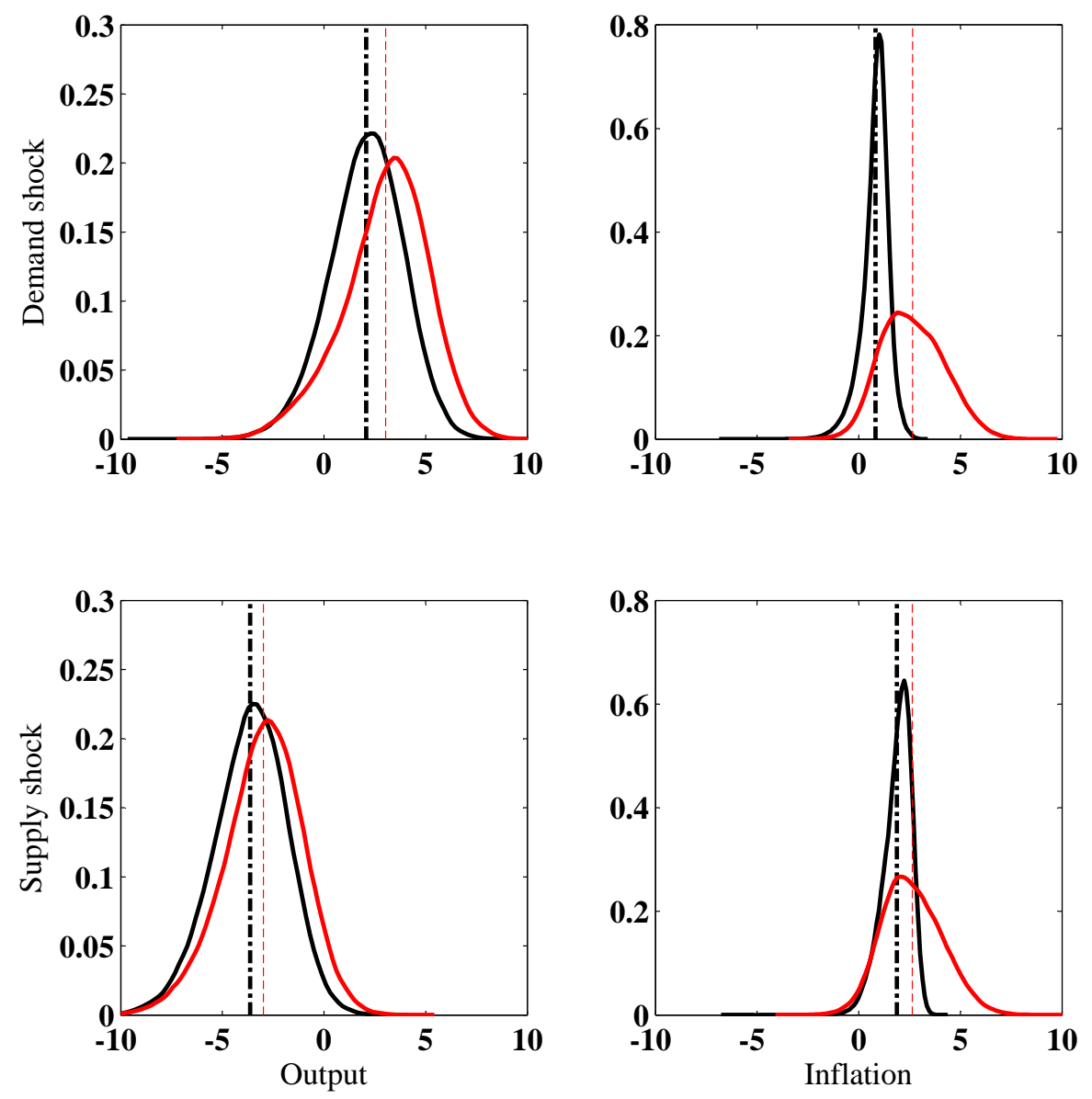

Figure 8: Distributions one period ahead when initial monetary regime is passive

Note: Red (resp. black) line plots the distribution of output and inflation one period after a 3 -standard deviation shock for endogenous (resp. exogenous) regime switching model. Initial monetary policy regime is passive. Distributions result from 100000 draws of future shocks and regimes and are approximated using a standard kernel estimate. The vertical red and black lines report the mean of the distributions. 


\section{References}

Abraham, R., J. Marsden, and T. Ratiu (1988): "Manifold Tensor Analysis, and applications," Applied Mathematical Sciences, 75.

Barthélemy, J. And M. Marx (2012): "Generalizing the Taylor Principle: New Comment," Working papers 403, Banque de France.

Bianchi, F. (2013): "Regime Switches, Agents' Beliefs, and Post-World War II U.S. Macroeconomic Dynamics," Review of Economic Studies, forthcoming.

Blake, A. P. And F. Zampolli (2006): "Optimal monetary policy in Markov-switching models with rational expectations agents," Bank of England working papers 298, Bank of England.

Blanchard, O. And C. M. Kahn (1980): "The Solution of Linear Difference Models under Rational Expectations," Econometrica, 48.

Calvo, G. A. (1998): "Capital Flows and Capital-Market Crises: The Simple Economics of Sudden Stops," Journal of Applied Economics, 0, 35-54.

Cно, S. (2009): "Characterizing Markov-Switching Rational Expectations Models," mimeo, School of Economics, Yonsei University.

Christiano, L. J. And J. D. M. Fisher (2000): "Algorithms for solving dynamic models with occasionally binding constraints," Journal of Economic Dynamics and Control, 24, $1179-1232$.

Clarida, R., J. Galí, and M. Gertler (2000): "Monetary Policy Rules And Macroeconomic Stability: Evidence And Some Theory," The Quarterly Journal of Economics, 115, $147-180$.

Coe, P. J. (2002): "Financial Crisis and the Great Depression: A Regime Switching Approach," Journal of Money, Credit and Banking, 34, 76-93.

Costa, O., M. Fragoso, and R. Marques (2005): Discrete-Time Markov Jump Linear Systems, Springer.

DAVIG, T. AND T. DoH (2008): "Monetary policy regime shifts and inflation persistence," Tech. rep.

Davig, T. And E. M. Leeper (2007): "Generalizing the Taylor Principle," American Economic Review, 97, 607-635.

(2008): "Endogenous Monetary Policy Regime Change," NBER International Seminar on Macroeconomics 2006.

Farmer, R. E. A., D. F. Waggoner, and T. Zha (2007): "Understanding the NewKeynesian Model when Monetary Policy Switches Regimes," NBER Working Papers. 
(2009a): "Indeterminacy in a forward-looking regime switching model," International Journal of Economic Theory, 5, 69-84.

(2009b): "Understanding Markov-switching rational expectations models," Journal of Economic Theory, 144, 1849-1867.

(2010a): "Generalizing the Taylor Principle: a comment," American Economic Review.

(2010b): "Minimal State variable solutions to Markov-Switching rational expectations models," to appear in Journal of Economic Dynamics and Control.

Fernández-Villaverde, J., P. Guerrón-Quintana, and J. F. Rubio-Ramírez (2010): "Reading the recent monetary history of the United States, 1959-2007," Review, 311-338.

Filardo, A. J. (1994): "Business-Cycle Phases and Their Transitional Dynamics," Journal of Business and Economic Statistics, 12, 299-308.

Filardo, A. J. And S. F. Gordon (1998): "Business cycle durations," Journal of Econometrics, 85, 99-123.

Foerster, A., J. Rubio-Ramirez, D. Waggoner, And T. Zha (2011): "Essays on Markov-Switching Dynamic Stochastic General Equilibrium Models," Foerster's PhD Dissertation, Chapter 2, Department of Economics, Duke University.

Hamilton, J. D. (1989): "A New Approach to the Economic Analysis of Nonstationary Time Series and the Business Cycle," Econometrica, 57, 357-84.

JIN, H. AND K. JUdD (2002): "Perturbation methods for general dynamic stochastic models," Working Paper, Stanford University.

Justiniano, A. And G. E. Primiceri (2008): "The Time-Varying Volatility of Macroeconomic Fluctuations," American Economic Review, 98, 604-41.

Kim, C.-J., J. Piger, And R. Startz (2003): "Estimation of Markov regime-switching regression models with endogenous switching," Journal of Econometrics, 143, 263-273.

KLEIN, P. (2000): "Using the generalized Schur form to solve a multivariate linear rational expectations model," Journal of Economic Dynamics and Control, 24, 1405-1423.

Leeper, E. M. And T. Zha (2003): "Modest policy interventions," Journal of Monetary Economics, 50, 1673-1700.

Liu, Z., D. F. Waggoner, And T. Zha (2010): "Sources Of Macroeconomic Fluctuations: A Regime-Switching Dsge Approach," Emory Economics 1002, Department of Economics, Emory University (Atlanta). 
Lubik, T. A. And F. Schorfheide (2004): "Testing for Indeterminacy: An Application to U.S. Monetary Policy," American Economic Review, 94, 190-217.

Mendoza, E. G. And V. Z. Yue (2011): "A General Equilibrium Model of Sovereign Default and Business Cycles," IMF Working Papers 11/166, International Monetary Fund.

Rothert, J. (2009): "Endogenous Regime Switching," mimeo.

Sims, C. A. (1982): "Policy Analysis with Econometric Models," Brookings Papers on Economic Activity, 13, 107-164.

Sims, C. A. And T. Zha (2006): "Were There Regime Switches in U.S. Monetary Policy?" American Economic Review, 96, 54-81.

Svennson, L. And N. Williams (2009): "Optimal Monetary Policy under Uncertainty in DSGE Models: A Markov Jump-Linear-Quadratic Approach," Central Banking, Analysis, and Economic Policies Book Series, Monetary Policy under Uncertainty and Learning, Klaus Schmidt-Hebbel and Carl E. Walsh and Norman Loayza (Series Editor), 13.

Woodford, M. (1986): "Stationary Sunspot Equilibria: The Case of Small Fluctuations around a Deterministic Steady State," mimeo.

- (2003): "Interest and prices: Foundations of a theory of monetary policy," Princeton University Press. 